ARTICLE

\title{
An epigenetic gene silencing pathway selectively acting on transgenic DNA in the green alga Chlamydomonas
}

\author{
Juliane Neupert ${ }^{1}$, Sean D. Gallaher (1) 2, Yinghong Lu' ${ }^{1,4}$, Daniela Strenkert ${ }^{1,2,5}$, Na'ama Segal ${ }^{1}$, \\ Rouhollah Barahimipour (10) 1, Sorel T. Fitz-Gibbon (10) ${ }^{3}$, Michael Schroda (1) ${ }^{1,6}$, Sabeeha S. Merchant (1) ${ }^{2,5}$ \& \\ Ralph Bock (iD) ${ }^{1 \times}$
}

Silencing of exogenous DNA can make transgene expression very inefficient. Genetic screens in the model alga Chlamydomonas have demonstrated that transgene silencing can be overcome by mutations in unknown gene(s), thus producing algal strains that stably express foreign genes to high levels. Here, we show that the silencing mechanism specifically acts on transgenic DNA. Once a permissive chromatin structure has assembled, transgene expression can persist even in the absence of mutations disrupting the silencing pathway. We have identified the gene conferring the silencing and show it to encode a sirtuin-type histone deacetylase. Loss of gene function does not appreciably affect endogenous gene expression. Our data suggest that transgenic DNA is recognized and then quickly inactivated by the assembly of a repressive chromatin structure composed of deacetylated histones. We propose that this mechanism may have evolved to provide protection from potentially harmful types of environmental DNA.

\footnotetext{
${ }^{1}$ Max-Planck-Institut für Molekulare Pflanzenphysiologie, Am Mühlenberg 1, D-14476 Potsdam-Golm, Germany. ${ }^{2}$ University of California Los Angeles, Department of Chemistry and Biochemistry, and Institute for Genomics and Proteomics, 607 Charles E. Young Dr. East, Los Angeles, CA 90095, USA.

${ }^{3}$ University of California Los Angeles, Department of Molecular, Cell and Developmental Biology, 610 Charles E. Young Dr. South, Los Angeles, CA 90095, USA. ${ }^{4}$ Present address: Nanjing University of Science and Technology, Institute of Chemical Biology and Advanced Materials, Xiaolingwei Street 200 , 210094 Nanjing, China. ${ }^{5}$ Present address: University of California Berkeley, Departments of Molecular and Cell Biology and Plant and Microbial Biology,

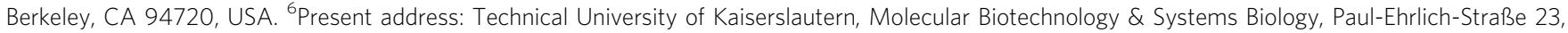
67663 Kaiserslautern, Germany. ${ }^{凶}$ email: rbock@mpimp-golm.mpg.de
} 
T he unicellular ciliated green alga Chlamydomonas reinhardtii belongs to the plant lineage Viridiplantae. It has become an invaluable reference system for nearly all areas of plant biology and, in addition, for medical research on ciliary diseases in humans ${ }^{1}$. The alga also provides an attractive production host for recombinant proteins, biofuels and green chemicals $s^{2-5}$. Although Chlamydomonas is readily transformable, transgene expression from the nuclear genome is notoriously inefficient $^{6,7}$ and, moreover, often unstable in that loss of transgene expression occurs with time ${ }^{8,9}$. A number of strategies have been pursued to overcome this serious limitation ${ }^{10}$, including the construction of hybrid promoters ${ }^{11}$, the inclusion of endogenous introns in the expression cassette ${ }^{12}$ and codon optimization of the foreign gene ${ }^{6,13,14}$. Although these strategies alleviated the problem to some extent in some cases, the expression of even standard transgenes (like the fluorescent reporters GFP and YFP) has remained very challenging. A more general solution has emerged from mutant screens for strains that show improved transgene expression properties. Two such mutant strains, UVM4 and UVM11, were isolated from a UV mutagenesis screen ${ }^{15}$ and have become widely used in cell biology studies and as tools for highlevel transgene expression ${ }^{16-19}$. Enhanced transgene expression correlates with greatly increased transcript levels, suggesting that the strains harbor mutations in gene(s) that cause strong epigenetic transgene silencing in the wild type ${ }^{15}$.

Here we report the identification of the gene underlying the transgene expression phenotype in the Chlamydomonas expression strains UVM4 and UVM11. We show that the transgenesilencing pathway specifically affects exogenously introduced DNA. Identification of a histone-modifying enzyme as the key factor for silencing to occur suggests that lack of coverage with histones is the distinguishing feature that allows cells to recognize transgenic DNA and inactivate it. The silencing mechanism identified in this work also highlights strategies how transgene expression can be improved in recalcitrant species.

\section{Results}

Transgenic DNA is associated with transcriptionally active chromatin in UVM4 and UVM11. When transformed with the YFP reporter gene, strong protein accumulation is readily detectable as a very bright yellow fluorescence signal in the cytoplasm of the UVM4 and UVM11 strains (Fig. 1a). By contrast, the transformed wild-type strain (CC-4350 alias cw15-302) and the wild-type-like control strain that was used for the UV mutagenesis screen (Elow47) do not show detectable YFP fluorescence. To confirm the assumed involvement of chromatin structure in mediating the high transgene expression capacity in strains UVM4 and UVM11 (ref. ${ }^{14}$ ), chromatin immunoprecipitation (ChIP) experiments were conducted. Using antibodies against different histone modifications with known associations to chromatin states in Chlamydomonas, the chromatin state at the transcriptional start site (TSS) of the transgene was assessed. Nucleosome occupancy was determined with antibodies against unmodified histone $\mathrm{H} 3$, active chromatin was probed with antibodies against acetylated histones H3 (K9/K14) and $\mathrm{H} 4$ (K5/K8/K12/K16), and repressive chromatin formation was assessed with antibodies against monomethylated lysine at position 9 of histone 3 (H3K9mel; refs. ${ }^{20,21}$ ).

For the ChIP analysis, large pools of independent YFP transformants of each strain were used to compensate for position effects and other types of variation in the chromatin that are related to the transgene integration sites in the genome (see "Methods"; Fig. 1b-e). It was shown previously that, when more than 240 transformants harboring the ble gene were pooled, variation in ble expression between individual transformants was averaged out ${ }^{22}$. We, therefore, generated two independent pools of more than 1200 transformants in each strain background and analyzed these pools in individual ChIP experiments (Fig. 1b-e). The DNA contents of ChIPed samples were determined by triplicate quantitative real-time PCR using primers spanning the TSSs. For the euchromatic histone marks (H3ac: acetylated histone 3, and H4ac: acetylated histone 4), ChIPed DNA fragments were normalized to values obtained for the endogenous CYC6 gene encoding cytochrome $c_{6}$ (as internal standard). CYC6 is only transcribed under copper limitation ${ }^{21}$ and, therefore, its chromatin state is expected to be unaltered in our strains and culture conditions. A telomere-flanking region (TFR) was shown to be associated with $\mathrm{H} 3 \mathrm{~K} 9 \mathrm{mel}$ in Chlamydomonas and was, therefore, chosen to normalize ChIPed DNA in our samples with an antibody targeting H3K9me1 (ref. ${ }^{21}$ ). In addition to the TSS region of the YFP transgene, which is driven by a copy of the endogenous PSAD promoter, we also determined the chromatin status of the two strong, endogenous RBCS2 and PSAD promoters, and the TSS region of the emetine resistance marker CRY1-1 (driven by a copy of the RBCS2 promoter sequence; vector pJN4) that had been introduced into the genome of the mutagenized strain Elow47 and served as read-out of transgene expression strength in the initial genetic screen ${ }^{15}$.

ChIP analysis with antibodies against the unmodified Cterminus of the core histone $\mathrm{H} 3$ showed that nucleosome occupancy is reduced at the transgenic TSS region (PSAD promoter upstream of YFP) in strains UVM4 and UVM11 (Fig. 1b). This finding indicates a more open chromatin structure and is consistent with increased transgene expression in UVM4 and UVM11. When specific histone marks associated with either transcriptionally active (H3ac and $\mathrm{H} 4 \mathrm{ac}$ ) or repressive (H3K9me1) chromatin were investigated, strains UVM4 and UVM11 displayed much higher levels of acetylated histones 3 and 4 at the transgenic TSS regions of both YFP and CRY1-1 (Fig. 1c, d), indicating the formation of transcriptionally active chromatin at these loci. At the same time, the transgenic TSS regions are associated with significantly lower levels of the repressive histone mark H3K9me1 in UVM4 and UVM11 than in strain Elow47 (Fig. 1e), suggesting that the formation of repressive chromatin at the integrated nuclear transgenes is reduced as a consequence of the mutations in UVM4 and UVM11. Notably, no differences in histone occupancy or histone modifications were detectable at the TSS region of the endogenous RBCS2 and PSAD genes, demonstrating that the changes of the chromatin state at the transgenic TSS regions have no effect on the chromatin state at the TSS of the corresponding endogenous promoter copies.

We previously showed that the loss of the transgene-suppression mechanism confers no obvious selective disadvantage to the UVM strains ${ }^{15}$. To test whether epigenetically controlled processes are deregulated in the mutants, we investigated whether transposons are released from suppression. To analyze copy numbers and insertion sites, Southern blot analyses were performed with mutant strains that had been continuously propagated for two years after their isolation. Two elements were investigated: TOC1 as a retrotransposon-like element (class I), and Gulliver as representative of a cut-and-paste DNA transposon (class II). No changes were detected in the mutant strains in comparison to Elow47, indicating that the epigenetic regulation of transposon activity is not affected by the mutation in the transgene-suppression pathway (Fig. 1f). Similarly, sensitivity to the DNA-damaging agent zeocin was unaffected by the mutations in UVM4 and UVM11 (Supplementary Fig. 1), and both global histone occupancy and specific histone modification levels were unaltered (Fig. 1g; Supplementary Fig. 2). Together, these data suggest that the mutations in UVM4 and UVM11 affect a pathway that specifically determines chromatin structure at new genomic loci that originate from the uptake and integration of exogenous DNA. We also showed that the silencing 
a
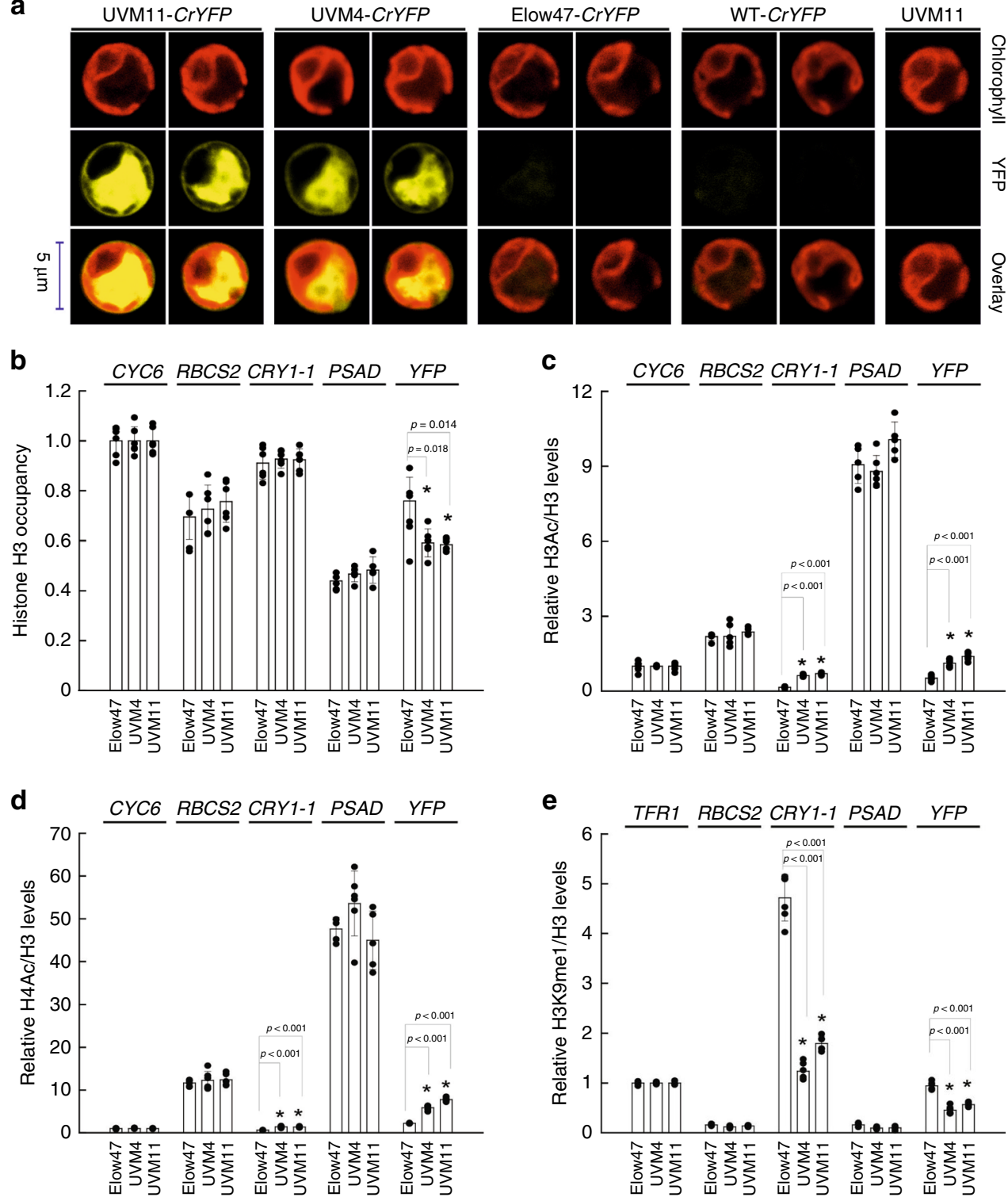

f
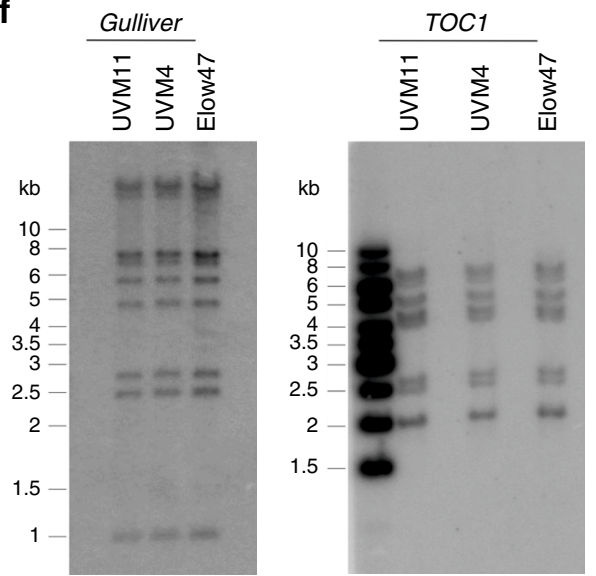

g

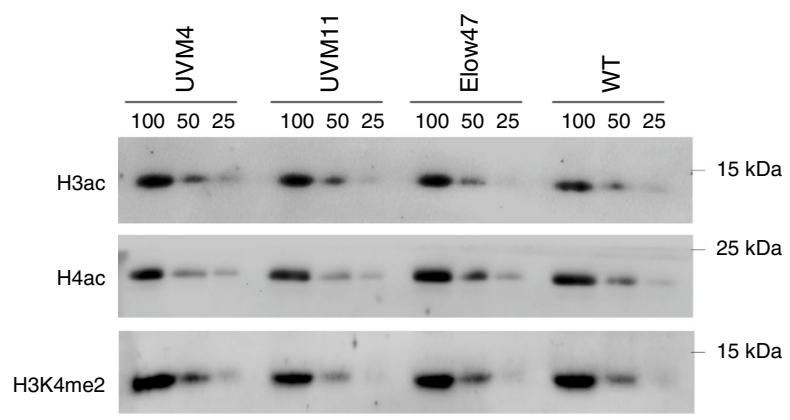

was not affected by the methylation status of the incoming DNA in that unmethylated DNA isolated from $\mathrm{dam}$ - and $\mathrm{dcm}$ - methylationdeficient bacterial strains was silenced equally efficiently. This is consistent with previous findings that also PCR-amplified DNA undergoes efficient silencing ${ }^{21}$.
Identification of the UVM11 and UVM4 mutations. To identify the mutations that cause the expression phenotype of the mutant strains, we sought to pursue a map-based cloning approach ${ }^{23}$. From a cross of the (cell wall-deficient, non-motile) strain UVM11 with the wild-type strain CC-124, individual segregants 
Fig. 1 Properties of Chlamydomonas expression strains. a YFP expression capacity in the expression strains UVM4 and UVM11 and the control strains Elow47 and CC-4350 (WT). All strains were transformed with the CrYFP construct ${ }^{14}$ and analyzed by confocal laser-scanning microscopy. For each strain, two independent CrYFP transformants were chosen that are representative of the strength of transgene expression. Chlorophyll fluorescence, YFP fluorescence and the overlay of the two fluorescences are shown. The non-transformed strain UVM11 served as negative control. Cells from at least 20 independently transformed colonies were individually analyzed by fluorescence microscopy (at least 200 cells per colony). The images show representative cells of each strain. b-e Comparative analysis of (b) histone $\mathrm{H} 3$ occupancy, (c) histone $\mathrm{H} 3$ acetylation, (d) histone $\mathrm{H} 4$ acetylation, and (e) histone $\mathrm{H} 3$ monomethylation at lysine 9 (H3K9me1) at the transcriptional start site (TSS) region of the endogenous genes CYC6, RBCS2 and PSAD and the transgenes CRY1_1 and YFP in the expression strains (UVM4 and UVM11) and their progenitor strain Elow47. Error bars indicate the standard error of six replicates. ${ }^{*} p<0.05$ (one-way ANOVA, Holm-Sidak post hoc test). Shown are averages and standard deviations of six ChIP experiments (individual data points shown as black dots; three experiments each were done on two independent pools of at least 1200 transformants). Values for each TSS region investigated were normalized to those obtained for the native CYC6 promoter (b-d) or a telomere-flanking region (TFRT; e). $\mathbf{f}$ Southern blot analysis to determine the activities of the DNA transposon Gulliver and the retrotransposon TOC1, 2 years after the isolation of the expression strains UVM4 and UVM11 from mutagenesis of Elow47. Total DNA was digested with Hindlll and probed for Gulliver (left panel) or digested with Hincll and probed for TOC1 (right panel). Fragment sizes of the molecular weight marker are given at the left in kb. Results from this experiment (performed once) were confirmed by transposon analysis on whole-genome sequencing data (Table 2; Supplementary Data 8) $\mathbf{g}$ Immunoblot analyses to determine the levels of acetylated histone 3 ( $\mathrm{H3ac}$ ), acetylated histone 4 ( $\mathrm{H} 4 \mathrm{ac})$, and dimethylated lysine 4 of histone $3(\mathrm{H} 3 \mathrm{~K} 4 \mathrm{me} 2)$ in the expression strains (UVM4 and UVM11) and the control strains (Elow47, and CC-4350 as WT). Total soluble protein was separated by denaturing SDS-PAGE, blotted and probed with specific antibodies as indicated. For each strain, a dilution series (100\%, 50\%, and $25 \%$ corresponding to 10,5 , and $2.5 \mu$ g total soluble protein, respectively) of the protein sample was loaded. Molecular weights were deduced from co-migrating protein markers and are given at the right in kDa. Each immunoblot experiment was performed at least twice with similar results obtained. Source data underlying Fig. $1 \mathrm{~b}-\mathrm{g}$ are provided as a Source Data file.

were screened for their capacity to express fluorescent reporter proteins to high levels. Strain UVM11-CW $(m t+)$ was isolated that shows the same strong transgene expression capacity as the UVM11 parental strain, but had regained the cell wall properties and motility of the wild-type. UVM11-CW $m t+$ was crossed to the interfertile polymorphic strain CC-2290 (alias S1-D2) mt(https://www.chlamycollection.org/). To be able to follow the segregation of the expression-conferring mutant alleles, a YFP transgene was introduced into the UVM11-CW $m t+$ strain. However, we noticed that, frequently, YFP fluorescence was retained in the entire progeny that possessed the YFP gene, despite the outcrossing of the expression-conferring mutation. This observation raised the possibility that, once a permissive chromatin structure has been established at the transgenic locus, the mutation in UVM11 (hereinafter referred to as uvm allele) is no longer needed to maintain active transgene expression. Moreover, this finding precluded the use of the YFP reporter as a phenotypic marker and, instead, necessitated phenotyping of the progeny of all crosses by transformation (i.e., transgene introduction). Thus, the individual segregants of all crosses had to be transformed with YFP to assess transgene expression capacity in the progeny (Fig. 2a; Supplementary Table 1; Supplementary Data 1 and 2). In total, 162 segregants were transformed at least once, and on average 20 transformed clones per segregant were screened for YFP expression, resulting in the analysis of $>3240$ transgenic clones. Subsequently, 48 segregants showing strong transgene expression were genotyped by PCR using markerspecific primers (http://www.chlamycollection.org/products/ mapping-kits/), and recombination frequencies were calculated (Fig. 2b; Supplementary Table 2). A low recombination frequency of $<20 \%$ was observed for two markers (CNA83 and PF25; Fig. 2b) on chromosome 10 (linkage group 10, LGX), indicating that the $u v m$ gene resides in this region (Supplementary Data 3). The inclusion of additional markers positioned between CNA83 and PF25 (ACE6301 and FLU; Fig. 2c) confirmed this conclusion.

Next, we investigated all genes localized between the FLU and PF25 markers and identified those that are predicted to be involved in chromatin-related functions (Supplementary Data 4). We then created four new markers (X1, X2, X3, and X4) by designing primers derived from the $3^{\prime}$ ends of four of these genes (Cre10.g461750, Cre10.g462200, Cre10.g464264, and Cre10.g464750; Supplementary Data 4) and determined their linkage to $u v m$ (Fig. 2c). Absolute linkage (in that the entire progeny displayed the marker allele associated with the UVM11CW parent) was observed for markers X1 and X2 (Fig. 2c; Supplementary Table 2).

Sequencing of the two genes (including their promoters and terminators) in strain UVM11 revealed a C-to-T transition at position +2728 of gene Cre10.g462200. Sequencing of the loci in UVM4 detected a G-to-A transition at position +431 in the same gene (Fig. 3a), strongly suggesting that Cre10.g462200 represents the UVM gene. To further confirm this conclusion, the genomes of both UVM strains were sequenced. Since the strains originated from UV mutagenesis, the mutation load in their genomes was high (with over 200 novel mutations per strain), as expected (Supplementary Tables 3 and 4; Supplementary Data 5). However, the number of genes containing distinct non-synonymous mutations in both strains was low and locus Cre10.g462200 was identified as one of only two such loci (and the only one on chromosome 10; Supplementary Table 5).

Cre10.g462200/UVM encodes a $65 \mathrm{kDa}$ putative Sir2-type histone deacetylase (SRTA). The Sir2 domain of Chlamydomonas SRTA is highly similar to the Sir2 domains of histone deacetylases in other eukaryotes, including Arabidopsis (AtSRT1), humans (HsSIRT6) and yeast (ScHstlp; Fig. 3b). The Gly-to-Ser exchange at position 55 in strain UVM4 and the Pro-to-Leu exchange at position 222 in strain UVM11 both affect highly conserved residues in the catalytic domain (Fig. 3b). Structural alignment of the Sir2 domains of the wild-type SRTA and the mutated versions from UVM4 and UVM11 based on the 3D structure of the Sir2 domain of human SIRT6 (Supplementary Fig. 3) supports the predicted effect on the catalytic activity in the mutant strains. The mutation in UVM4 likely interferes with the binding of the cofactor $\mathrm{NAD}(+)$, while the UVM11 mutation is more likely to affect protein structure and/or the interaction with other proteins.

The Chlamydomonas genome contains 17 genes that are annotated as histone deacetylases (HDAC) and three of these are predicted to be of the Sir2-type (subsequently referred to as SRTA encoded by Cre10.g462200/UVM, SRTB encoded by Cre12.g524650 and SRTC encoded by Cre05.g234663; Supplementary Table 6). All other histone deacetylases (HDA1-HDA14) are homologous to the Arabidopsis histone deacetylases of the RPD3/HDA1 type ${ }^{24}$. Interestingly, Chlamydomonas does not possess any member of the HD2-family (type-2 HDACs), which is a plant-specific HDAC family ${ }^{25}$. A phylogenetic tree of all Chlamydomonas Sir2type histone deacetylases and sirtuins from representative model 
a
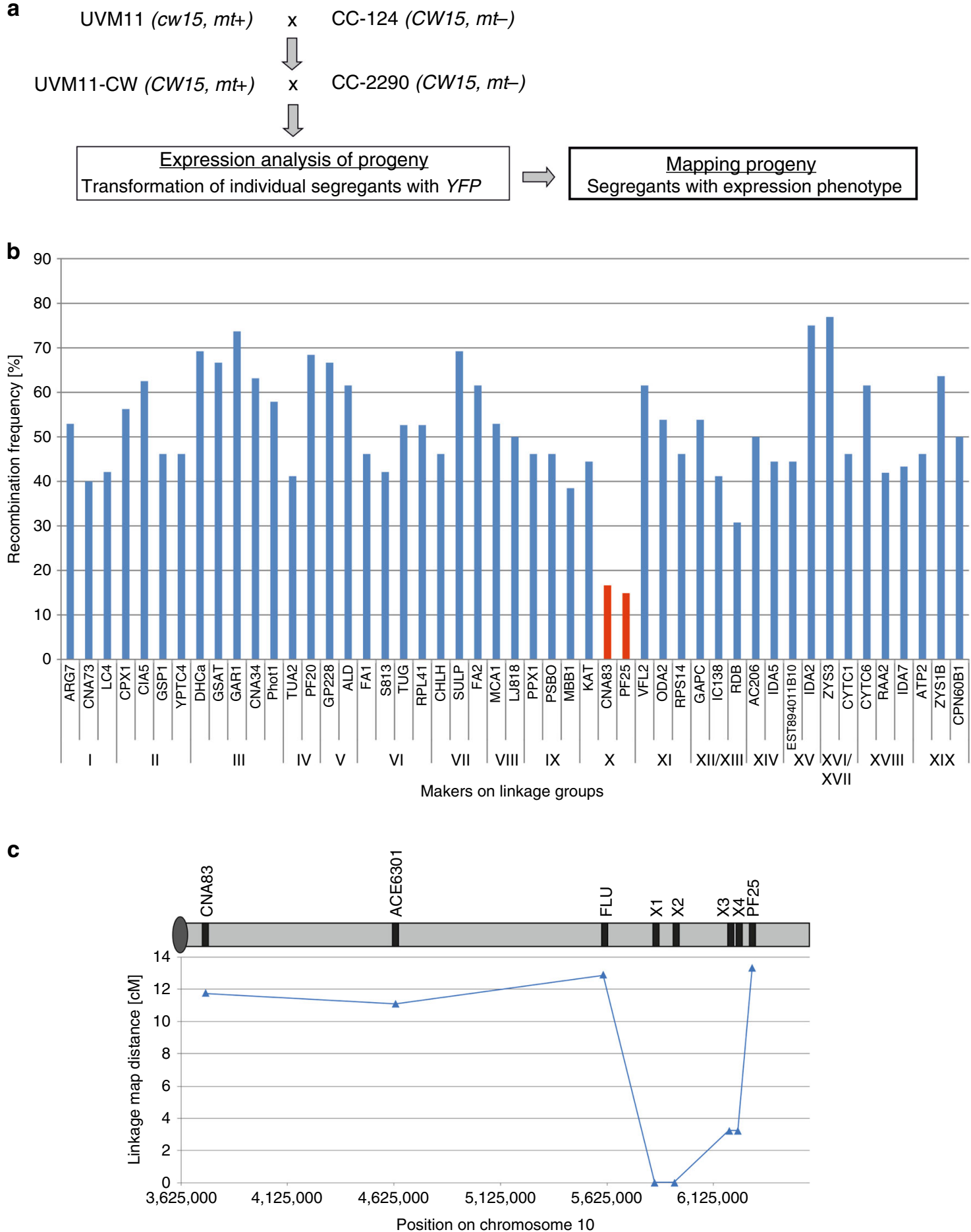

Fig. 2 Identification of the UVM gene. a Mapping strategy. The cell wall-deficient expression strain UVM11 was crossed to the wild-type strain CC-124 in order to reintroduce cilia (required for efficient mating). Offspring (UVM11-CW) that showed similarly strong transgene expression capacity as the parental UVM11 strain was then crossed to the polymorphic strain CC-2290. The progeny of this cross was phenotyped for transgene expression efficiency by transforming every segregant with the YFP reporter gene and analyzing on average 20 transformants per segregant. Offspring displaying high-level YFP expression was used as mapping population. $\mathbf{b}$ Marker analysis links the transgene expression phenotype to a locus on chromosome 10. The two markers showing low recombination frequency $(<20 \%)$ are indicated in red. c Fine mapping of uvm on chromosome 10 . The new markers X1, X2, X3, and X4 were derived from candidate genes that reside between markers CNA83 and PF25 and potentially have chromatin-related functions ${ }^{36}$ (http://phytozome.jgi. doe.gov/).

organisms revealed that SRTA is more closely related to the sirtuintype HDAC from Arabidopsis and humans than to SRTB and SRTC (Fig. 3c). In contrast to the Chlamydomonas histone deacetylases SRTB and SRTC, which group with the sequence-related phylogenetic sirtuin classes II and III, respectively, the Chlamydomonas histone deacetylase SRTA falls into class IV, a class that does not contain prokaryotic members (Fig. 3 c; ref. ${ }^{26}$ ). Class IV includes the human sirtuins SIRT6 and SIRT7 that are both nucleus- 
a
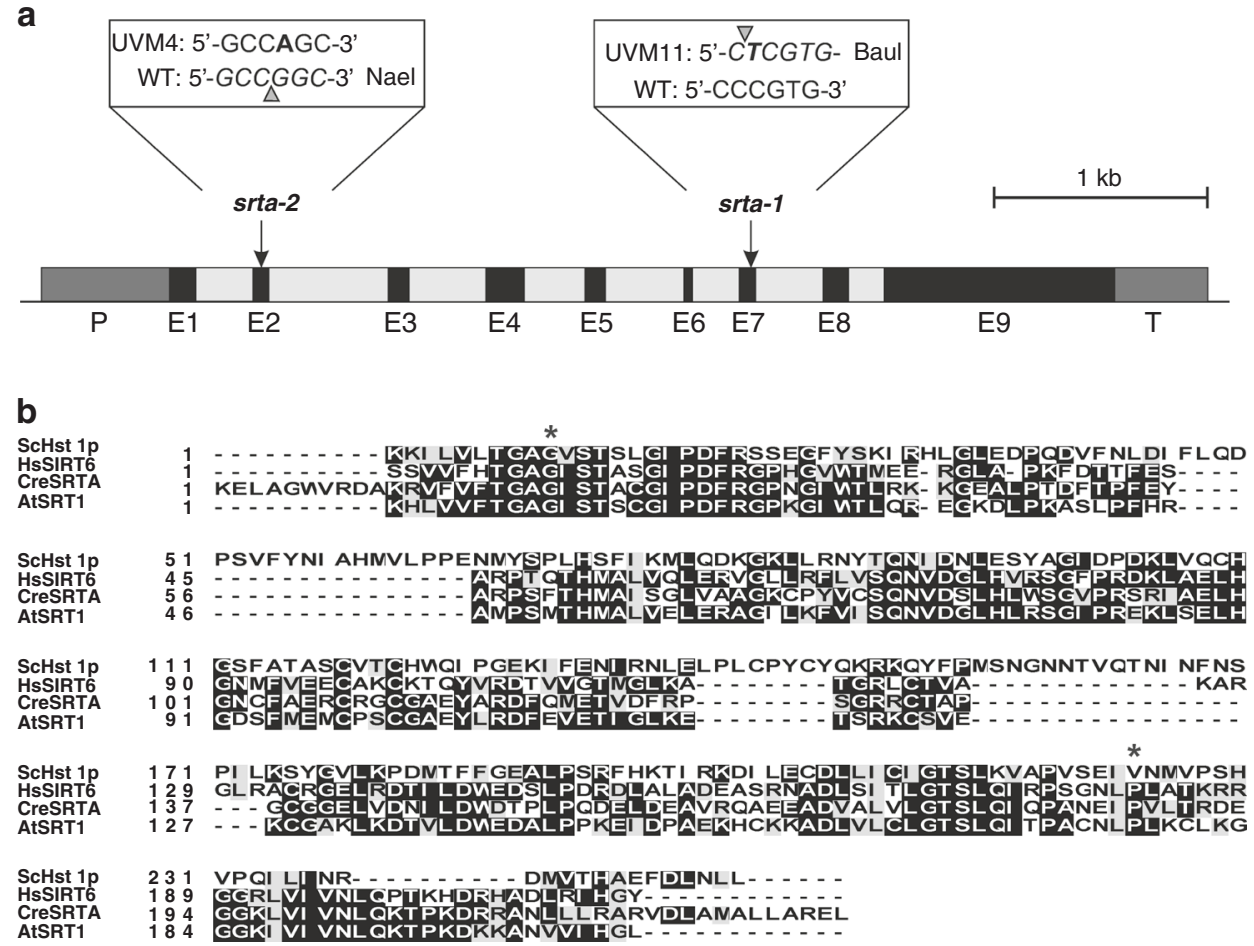

C

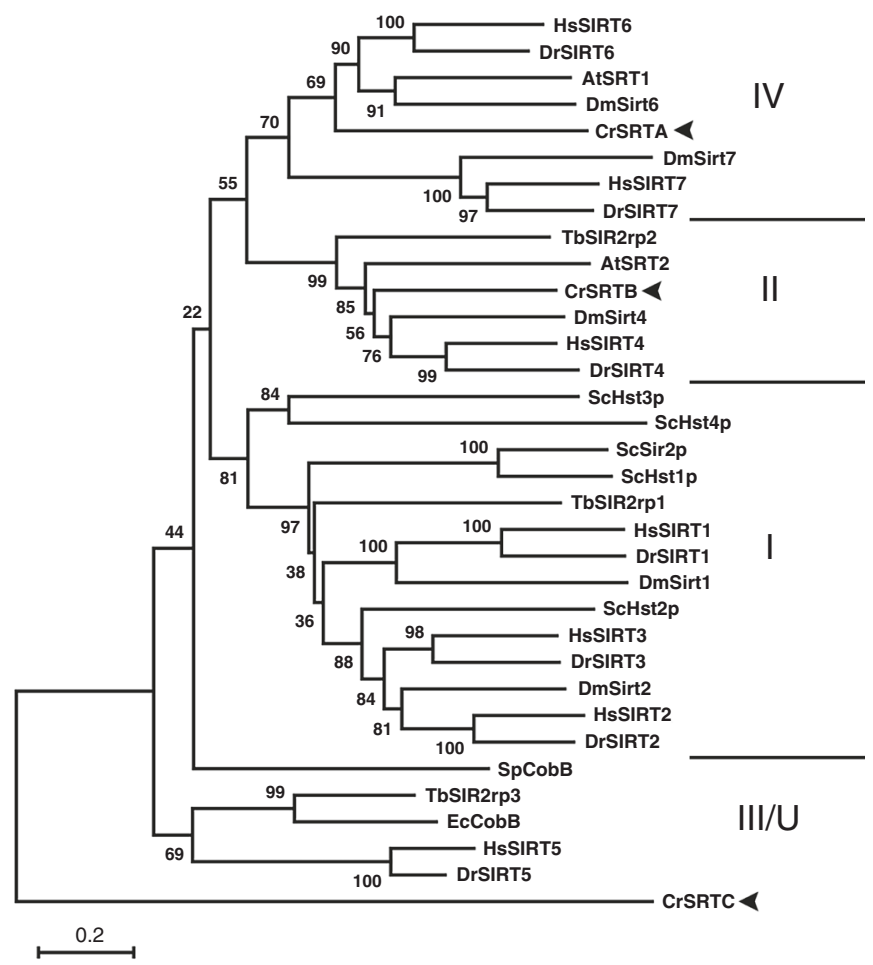

localized and play an important role in epigenetic regulation ${ }^{27,28}$. They catalyze histone deacetylation, but also act on various other cellular substrates, including DNA repair proteins, tumor necrosis factor alpha and a subunit of RNA polymerase I (refs. ${ }^{29-31}$ ).

Introduction of the SRTA wild-type allele restores poor transgene expression capacity. To test whether transgene silencing by the HDAC SRTA is genetically dominant over active transgene expression conferred by the srta mutant alleles of strain UVM11 (hereinafter referred to as srta-1) and strain UVM4 (hereinafter referred to as srta-2) and to confirm our assumption that srta-1 and srta-2 represent loss-of-function alleles, complementation analyses were undertaken. To this end, the wild-type cDNA sequence of SRTA and that of the srta-2 mutant allele were cloned in an expression cassette containing the native SRTA regulatory sequences (Fig. 4a). Both expression strains were then transformed with these constructs and, additionally, with a FLAG-tagged version of SRTA (Fig. 4a; Table 1). Transgenic clones were selected on hygromycin and the presence of the complete SRTA cassette was 
Fig. 3 Structure of the Cre10.g462200/UVM gene and properties of the encoded Sir2-like NAD( + ) dependent protein deacetylase. a Expression strains UVM4 and UVM11 each carry a non-synonymous point mutation in the coding region of the Cre10.g462200 gene. Exons (E1-E9) are shown as black, introns as light gray boxes. Promoter $(P)$ and terminator $(T)$ sequences are represented as dark gray boxes. Arrows indicate the positions of the point mutations in strain UVM4 (mutant allele srta-2) and strain UVM11 (mutant allele srta-1). The G-to-A transition in srta-2 leads to the loss of a Nael restriction site, the C-to-T transition in srta-1 leads to gain of a Baul restriction site. b Alignment of the Sir2 domain of Chlamydomonas SRTA with the Sir2 domains of its closest homologs from Arabidopsis (AtSRT1), humans (HsSIRT6) and yeast (ScHst1p). Residues conserved in more than 50\% of the sequences are shown in black boxes. Similar amino acids are shaded in gray. The residues affected by the two UVM mutations are marked by asterisks. c Phylogenetic tree of Sir2-type histone deacetylases from Chlamydomonas reinhardtii (CrSRTA-C) and sirtuins from other model organisms, including Saccharomyces cerevisiae (ScSir2p, ScHst1-4), Drosophila melanogaster (DmSirt1, 2, 4, 6, and 7), Danio rerio (DrSIRT1-7), Homo sapiens (HsSIRT1-7), Arabidopsis thaliana (AtSRT1 and AtSRT2), the protozoan Trypanosoma brucei (TbSIR2rp1-3), and the bacteria Escherichia coli (EcCobB) and Streptococcus pneumoniae ( $\mathrm{SpCobB}$ ). The tree is based on a ClustalW multiple sequence alignment and was conducted using the neighbor-joining method with 500 bootstrap replicates. The optimal tree with the sum of branch length $=16.60516448$ is shown. The percentages of replicate trees in which the associated taxa clustered together in the bootstrap test (500 replicates) are shown next to the branches. The tree is drawn to scale, with branch lengths in the same units as those of the evolutionary distances used to infer the phylogenetic tree. The evolutionary distances were computed using the Poisson correction method, and both the ClustalW MSA and the Neighbor-Joining phylogenetic analysis were conducted with the MEGAX software (Molecular Evolutionary Genetics Analysis, https://www.megasoftware.net/mega4/). The Sir2 homologs found in Chlamydomonas are marked with black arrows. The sequencebased phylogenetic classes of sirtuins ${ }^{26}$ are given at the right (I, II, III, IV, and U).

assessed by PCR (for all pJR81 transformants with primers X2-fw and M13uni; Supplementary Table 10). Subsequently, the transgenic strains were supertransformed with the YFP reporter to determine their transgene expression phenotype. (The term supertransformation refers to an already transgenic strain that is transformed with another construct.) Supertransformants were selected on paromomycin and, for each SRTA transformant, $\geq 20$ supertransformants were analyzed by fluorescence microscopy. While the transformation of UVM4 and UVM11 produced YFP-expressing clones at high frequency (Supplementary Fig. 5), strains that had been transformed with the complementation constructs (pJR81 and pJR88; Fig. 4), produced much lower numbers of YFP-expressing clones (YET: YFP-expressing transformants; as in our mapping strategy, YET values of $\geq 30 \%$ were scored as transgene expression phenotype; Table 1; Supplementary Figs. 4 and 5). Importantly, the complementation efficiency correlated with the expression strength of the transgenically introduced SRTA wild-type allele, in that the complemented clones displaying a strong expression of the wild-type allele entirely or nearly entirely lost their transgene expression capacity (Fig. 4b). Complementation was also achieved with the FLAG-tagged SRTA sequence (Fig. 4a; Table 1), indicating that the C-terminal tag is compatible with protein function. Quantitation of YFP fluorescence in randomly selected YFP supertransformants confirmed the loss of YFP expression capacity in the complemented strains (Supplementary Fig. 5). As an additional control, the UVM4 and UVM11 strains were transformed with the mutated allele srta-2 (pJR85; Fig. 4a). As expected, this did not result in complementation of the expression phenotype of the strains, as revealed by supertransformation with the YFP cassette (Supplementary Table 7).

Finally, we also obtained an insertion mutant (LMJ.RY0402. 148523) from the Chlamydomonas Library Project $\left(\mathrm{CLiP}^{32}\right)$ that carries a predicted insertion in the SRTA locus. The mutant, hereafter named srta-3, turned out to harbor a rearrangement involving two loci: Cre10.g462200 on chromosome 10 (encoding SRTA) and Cre03.g200350 on chromosome 3 (encoding SMM12, a putative demethylmenaquinone methyltransferase), with the $5^{\prime}$ junction being in SRTA and the $3^{\prime}$ junction in SMM12 (Supplementary Figs. 6-8; Supplementary Table 8). Due to this potential complication, an insertion mutant in SMM12 was additionally analyzed (LMJ.RY0402.153656; ref. ${ }^{32}$; Supplementary Fig. 9). The srta-3 mutant does not accumulate any SRTA transcript (Supplementary Fig. 6b) and, therefore, represents an SRTA null allele. Determination of transgene expression efficiency by transformation with the YFP reporter gene confirmed that loss of
SRTA function results in the transgene expression phenotype (Supplementary Table 8). The smm12 mutant strains did not exhibit detectable YFP fluorescence, as expected.

Low sensitivity to the silencing of transgenes pre-integrated into the genome. The specific action of SRTA on transgenes raises the question how the enzyme specifically recognizes exogenous DNA sequences. A time point when transgenic DNA could be distinguished from endogenous DNA is prior to or early during the integration of the foreign DNA into the genome. This is because the foreign DNA enters the cell as naked DNA and, possibly, because the DNA repair machinery is recruited to the site of integration. It seems conceivable that the SRTA enzyme specifically acts on chromatin that is assembled de novo, while established chromatin structures are largely protected from its activity.

To test this hypothesis, we isolated several independent YFP transformants of strain UVM11 that displayed strong YFP expression $^{14,15}$, supertransformed them with the SRTA wild-type allele (or the empty vector as a control) and measured YFP fluorescence of several supertransformants. While there was a reduction of YFP fluorescence in some of the SRTA supertransformants (e.g., strains C, D and G in Fig. 5a), several transgenic strains displayed no detectable loss of YFP fluorescence in the supertransformants (e.g., strains A, B, and I in Fig. 5a).

From these data, we conclude that, once the transgene is stably integrated into the genome and a permissive chromatin state has been established, its expression status can become refractory to the action of the SRTA histone deacetylase. The degree of this insusceptibility may depend on the integration site of the transgene in the genome (Fig. 5a). To also test for meiotic stability of the loss of epigenetic transgene inactivation, we performed crosses of a YFP-carrying expression strain with a wild-type strain. After tetrad dissection, individual segregants were tested for YFP fluorescence and genotyped by PCR for the presence of the srta-1 point mutation and the YFP cassette (Fig. 5b, c). Consistent with our earlier observation that YFP expression cannot be used as a marker for mapping (see above), we observed a number of segregants that carried the SRTA wild-type allele, but yet expressed YFP (Fig. 5b, c). Thus, even in the absence of the srta-1 mutation, the YFP expression cassette can stay active. This was surprising, given that transgenes usually undergo very efficient silencing upon transformation of wild-type strains.

Treatments with histone deacetylase inhibitors do not stimulate transgene expression in the wild type. Having identified a 
a

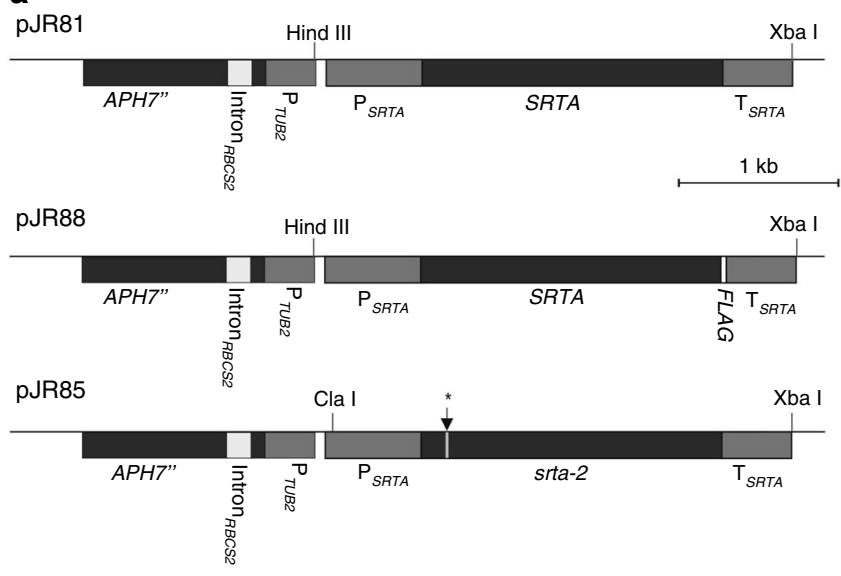

b

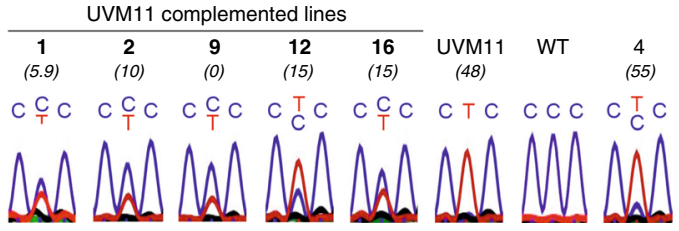

Fig. 4 Complementation of the uvm mutants with the wild-type SRTA

allele. a Physical map of the transformation vectors used for

complementation of strains UVM4 and UVM11. Exons are shown in black, introns in light gray, and promoter and terminator sequences in dark gray. Expression of the SRTA CDNA is driven by the native SRTA promoter $\left(P_{\text {SRTA }}\right), 5^{\prime} U T R$, and $3^{\prime} U T R\left(T_{\text {SRTA }}\right)$. The hygromycin resistance gene $A P H 7^{\prime \prime}$ under control of the TUB2 promoter was used as a selectable marker. Vector pJR88 additionally contains a FLAG-tag sequence fused to the SRTA coding region. pJR85 served as a control and carries the mutated srta-2 allele as found in strain UVM4 (location of the mutation indicated by the vertical arrow). b The complementation efficiency correlates with the expression strength of the transgenically introduced SRTA wild-type allele. Five independent SRTA transformants ( $p$ JR88) of strain UVM11 were assayed for expression of the endogenous srta-1 mutant allele relative to the SRTA wild-type allele introduced by the transformation. For each complemented line and control strain, the YFP transgene expression capacity is shown in parentheses (YET given in \%; cf. Table 1). The cDNA region harboring the srta-1 point mutation was amplified and sequenced. The height of the T vs. C peaks at the mutated position (nucleotide in the middle and indicated by two letters, with the top one denoting the dominant transcript) is a proxy of relative mRNA abundances. cDNA of UVM11 and the wild type-like strain Elow47 (WT) served as controls. Transformant number 4 that showed only very low SRTA transgene expression and, consequently, no complementation (YET $=55 \%$ ) was included as negative control. mutated histone deacetylase gene as the cause of the superior transgene expression capacity of the UVM4 and UVM11 strains, we next wanted to test if YFP transgene expression can be stimulated in wild-type strains by pharmacological inhibition of HDAC activity. To this end, we exposed YFP transformants of the wild type-like strain Elow47 before or after transformation to three different chemicals that are known to act as inhibitors of HDACs.

Application of the HDAC inhibitor trichostatin to YFP transformants of Elow47 and UVM11 led to an increase in total levels of acetylated histone 3 after $20 \mathrm{~h}$ of incubation with $200 \mathrm{ng} \mathrm{mL}^{-1}$ trichostatin (Supplementary Fig. 10), indicating that the drug is taken up and exerts its expected action. Despite the increase in H3ac levels, the treatment did not result in enhanced accumulation of YFP, neither in the Elow47 transformants (where the YFP transgenes remained silenced) nor in the transformant of the expression strain (where the YFP expression level remained unchanged). This result is in line with the fact that trichostatin does not inhibit HDACs of the sirtuin class ${ }^{33}$.

Sirtinol and related compounds have been described as potent inhibitors of HDACs of the sirtuin type ${ }^{33,34}$. When cultures of YFP transformants of strains Elow47 and UVM4 were incubated with different concentrations of sirtinol for $48 \mathrm{~h}$, none of the treatments resulted in higher levels of YFP accumulation (Supplementary Fig. 11). Also, total levels of acetylated histone H3 remained unchanged (Supplementary Fig. 11).

Finally, we tested the sirtuin inhibitor OSS_128167, which was reported to selectively block activity of SIRT6 in mammals ${ }^{35}$. SIRT6 is the closest human homolog of the Chlamydomonas SRTA protein, with both proteins belonging to the class IV of sirtuins (Fig. 3c). Treatment of algal cultures with OSS_128167 did not result in enhanced expression of the YFP transgene in three independent YFP transformants of Elow47 (Supplementary Fig. 12). Also, YFP accumulation could not be further boosted in a YFP transformant of UVM4 (Supplementary Fig. 12), indicating that the chromatin state of the transgene cannot be altered by the drug treatment.

It seems possible that OSS_128167 is not efficiently taken up by algal cells or does not inhibit the Chlamydomonas SRTA, although the human SIRT6 is more closely related to the Chlamydomonas SRTA than to the human members of sirtuin class I, SIRT1 and SIRT2 (Fig. 3c). Interestingly, SIRT1 and SIRT2 were shown to also be inhibited by OSS_128167, albeit to a much lesser extent (with $\mathrm{IC}_{50}$ values that are 10-20 times higher than for SIRT6; ref. ${ }^{35}$ ). However, treatment of YFP transformants of Elow47 with increased amounts of the inhibitor (200 $\mu$ M OSS_128167) also did not show any effect on YFP accumulation. Moreover, immunodetection of acetylated histone $\mathrm{H} 3$ versus total $\mathrm{H} 3$ revealed no increase in the levels of $\mathrm{H} 3 \mathrm{ac}$

Table 1 Introduction of the SRTA wild-type allele into UVM4 and UVM11 restores the poor transgene expression phenotype of
the wild type.
\begin{tabular}{llllll} 
Plasmid; strain & YET < $\mathbf{3 0}$ & YET = & YET $=\mathbf{1 - 1 0}$ & YET $=\mathbf{1 1 - 2 0}$ & YET = 21-29 \\
\hline pJR81; UVM4 & 69.2 & 11.5 & 34.6 & 7.7 & 15.4 \\
pJR81; UVM11 & 29.2 & 0.0 & 20.8 & 4.2 & 4.2 \\
pJR88; UVM4 & 38.9 & 5.6 & 5.6 & 16.7 & 11.1 \\
pJR88; UVM11 & 27.8 & 5.6 & 11.1 & 11.1 & 0.0
\end{tabular}

Transformants selected on hygromycin were analyzed for their gene expression capacity by supertransformation with the YFP reporter gene and analysis of $\geq 20$ supertransformants per line for YFP fluorescence. Complementation was assumed if $<30 \%$ of the supertransformants showed strong YFP expression. The transformants were grouped according to their transgene expression phenotype as YET (YFP Expressing Transformants) in percent (i.e., number of YFP-expressing transformants relative to the total number of transformants analyzed for each SRTA-transformed line). The first column gives the total complementation efficiency (YET $<30 \%$ ), the second column provides a breakdown into four complementation classes $(Y E T=0 \%$, YET $=1-10 \%, Y E T=11-20 \%$ and $Y E T=21-29 \%)$. Note that, in Chlamydomonas, the complementation efficiency is usually lower than $50 \%$, with a major reason being that typically, approximately half of the transformants have not integrated the complete expression cassette into their genome ${ }^{15}$. For further details, see Supplementary Fig. 4. 


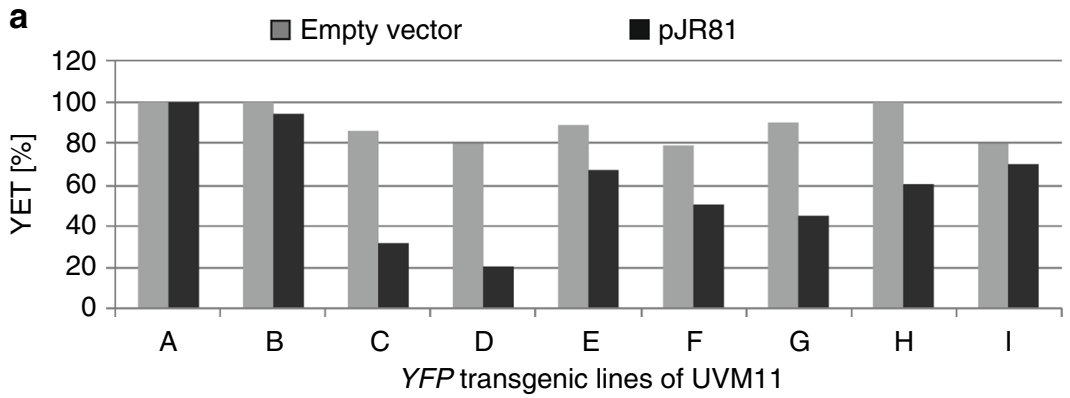

b

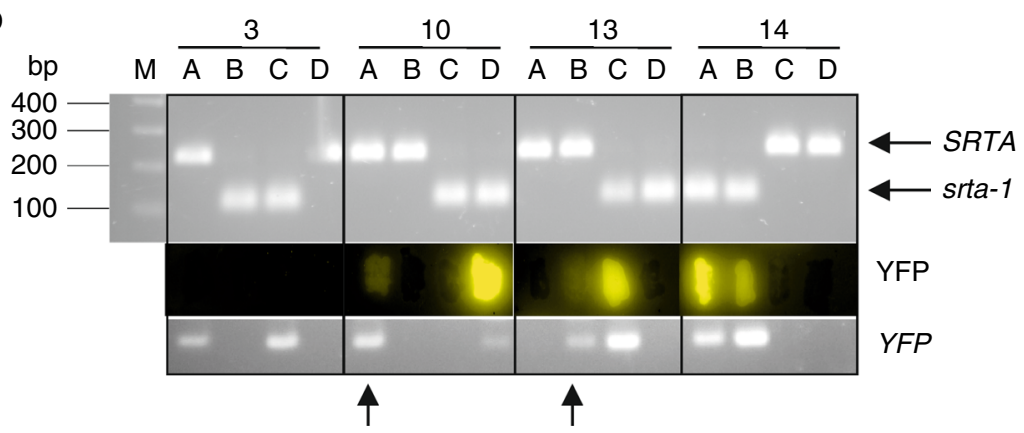

C

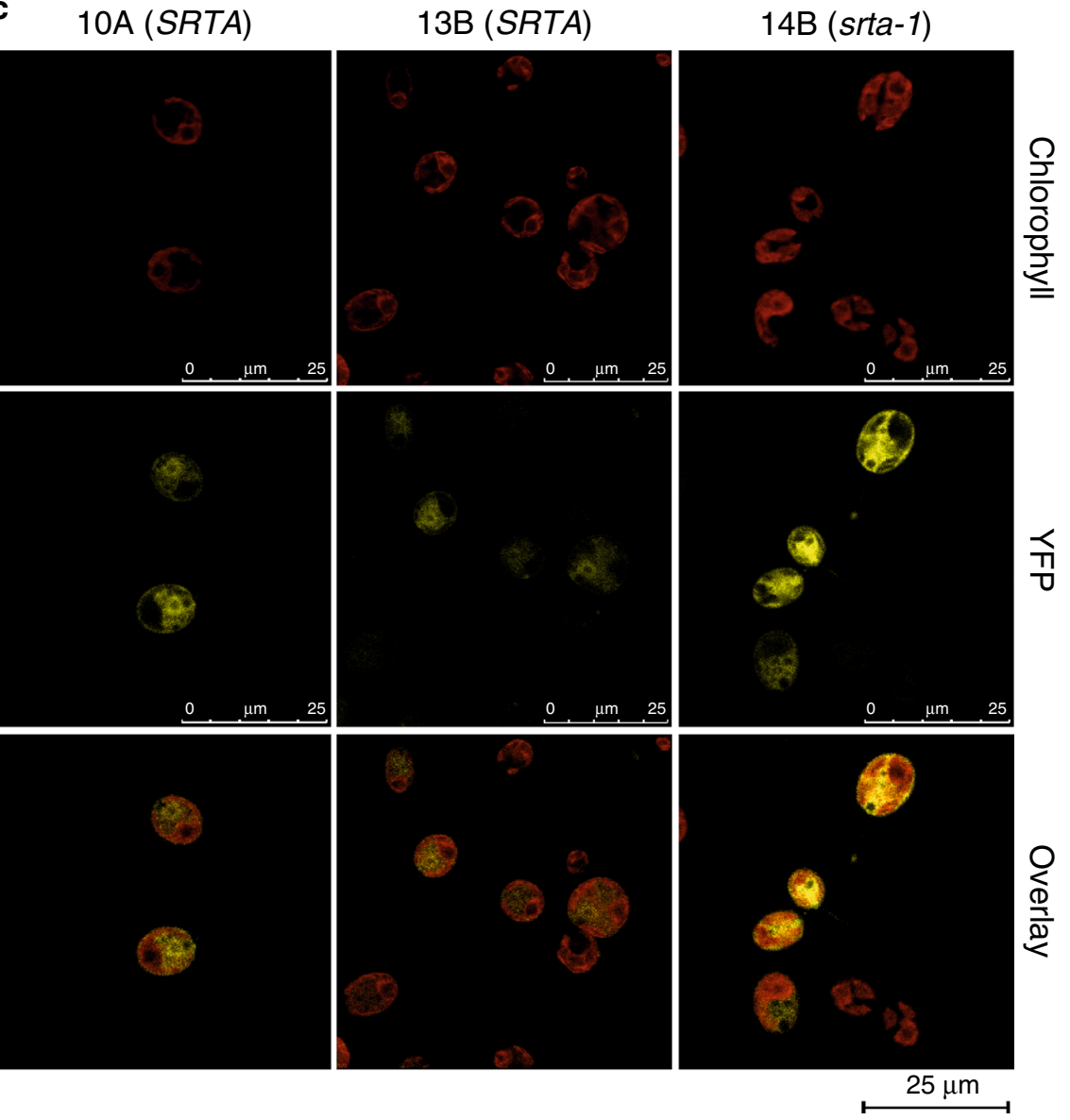

after 48 hours of OSS_128167 treatment (Supplementary Fig. 12). Thus, it remains possible that OSS_128167 does not inhibit the Chlamydomonas SRTA protein. Alternatively, it could inhibit SRTA, but SRTA affects neither global histone acetylation levels nor the chromatin status of transgenes that have already stably integrated into the genome.
The transcriptomes of the expression strains, the complemented strains, and their parental strain are very similar. To distinguish between a general role in transcriptional regulation and a possible transgene-specific action of the SRTA protein, we sought to identify endogenous genes that are affected by the srta mutations. To this end, the transcriptomes of both mutant strains 
Fig. 5 SRTA preferentially silences incoming naked DNA. a Complementation of srta-1 in YFP-expressing UVM11 strains by supertransformation with the SRTA wild-type allele. Several independent YFP transformants of UVM11 that showed strong YFP fluorescence were supertransformed with plasmid pJR81 (carrying the SRTA wild-type allele) and, on average, 20 supertransformants per strain were analyzed for YFP expression. As a control, the same number of supertransformants was generated with the empty vector and analyzed for YFP expression. $\mathbf{b}$ Epigenetic stability of YFP gene expression in meiosis. An expression strain carrying the srta-1 allele and expressing YFP to high levels was crossed to the wild-type strain CC-1690. Segregants of four tetrads were genotyped for the presence of the wild-type SRTA or the mutant (srta-1) allele by PCR with primers amplifying a fragment containing the mutation followed by digestion of the PCR amplicon with the restriction enzyme Baul, for which the point mutation in the srta-1 allele generates a new recognition site. Segregants were also genotyped for the YFP cassette, and YFP fluorescence was measured by scanning colonies grown on solid medium. Arrows mark segregants that carry the SRTA wild-type allele but nonetheless retain YFP fluorescence. c Confirmation of retention of YFP expression in the presence of the SRTA wild type-allele by confocal microscopy. Descendants of the crosses shown in (b) were analyzed and their genotype is given in parentheses. Results shown in (b) and (c) were obtained from a single crossing experiment, and similar results were obtained from crosses performed for the mapping analysis of uvm using strains UVM11-CW and CC-2290 (alias S1-D2). Source data underlying Fig. 5b are provided as a Source Data file.

(UVM4 and UVM11) were characterized and compared to the transcriptome of their parental strain, the wild type-like control strain Elow47 (RNAseq experiment batch 1). To better filter out differentially expressed genes (DEGs) that are caused by the UV light-induced background mutations in the genomes of UVM4 and UVM11, a second transcriptome analysis was performed in which UVM11 and two independently generated complemented strains of UVM11 were analyzed (RNAseq experiment batch 2). When the datasets from both RNAseq experiments were compared, only a very small percentage of the genes were found to be differentially upregulated or downregulated, with no more than $1.7 \%$ of the genes in any given strain showing differential expression (Fig. 6a; Supplementary Data 6 and 7). When a principal component analysis (PCA) on the 1000 genes that are the most variable from the dataset composed of batch 1 and batch 2 was performed, the by far largest principal component (PC1) identifies batch effects (although every effort was made to treat the two sets of samples identically). Only the third principal component (PC3), representing only $7.5 \%$ of the variance, corresponds to some extent with the SRTA allele status of the strains (Fig. 6b).

Interestingly, strains UVM4 and UVM11 showed only 168 and 193 DEGs in comparison to the control strain Elow47 (Fig. 6a,c). Of those, 151 and 174 genes were upregulated in UVM4 and UVM11, respectively, whereas 17 and 19 genes were downregulated. Strains UVM4 and UVM11 share 100 DEGs that are upregulated and 4 DEGs that are downregulated (Fig. 6c).

If there were any endogenous Chlamydomonas genes that are regulated by SRTA, then these genes should be among the class of upregulated genes in UVM4 and UVM11 as identified from our batch 1 RNAseq analysis and, additionally, should be among the class of downregulated genes in the complemented UVM11 strains compared to UVM11 as identified from our batch 2 RNAseq analysis. Remarkably, only 18 genes belong to this category (Fig. 6d; Supplementary Data 6 and 7). Among the 18 shared DEGs, only four genes had an annotation of gene function given and, therefore, no enrichment of specific classes of genes or gene functions could be observed. In addition, those gene candidates generally show rather low expression levels, with the majority having values of 1 FPKM or lower in most samples (Supplementary Data 6 and 7). Although it currently cannot be excluded that some of these genes are deregulated due to the loss of SRTA, their very low number (compared to the total number of protein-coding genes in the genome of Chlamydomonas which is more than 17,700; ref. ${ }^{36}$ ) excludes a general role of SRTA in chromatin modification and transcriptional regulation.

Interestingly, the transcript abundance of $S R T A$ is also unchanged in the mutant strains compared to the control strain (Supplementary Fig. 13), suggesting that the mutations in strains UVM4 and UVM11 do not affect mRNA half-life and, moreover, indicating that there is no compensation mechanism to the SRTA deficiency that would operate at the level of transcription or mRNA stability.
Prior to RNAseq analysis, both complemented strains (UVM11-pJR88-1 and UVM11-pJR88-9; cf. Fig. 4b), which had been generated four years ago and maintained on hygromycin-containing medium ever since, were re-analyzed for their complementation efficiency. Both strains still showed unaltered strong expression of the introduced SRTA wild-type allele, as determined from the ratio of sequenced SRTA versus srta cDNA amplicons as well as from the RNAseq data. A new supertransformation of the strains with the YFP cassette confirmed the functional complementation in that most of the supertransformed colonies showed no (or only very weak) YFP fluorescence. This result shows that the wild-type form of the SRTA enzyme does not repress its own expression by acting on the (transgenically introduced) SRTA genomic locus. Instead, normal-level SRTA expression is maintained and the SRTA enzyme acts only on newly introduced transgenic DNA, as evidenced by supertransformation with the YFP cassette. This finding lends further support to our hypothesis that, once the chromatin status of the transgene is established (prior to or upon integration into the genome), it remains remarkably stable over time.

In sum, the very small changes in the transcriptomes and the lack of evidence for specific effects of the mutations in SRTA on endogenous gene expression argue against a general role of SRTA in the regulation of nuclear gene expression in Chlamydomonas. These results are in line with our previous findings that, apart from the strong expression of foreign genes, there is no other detectable phenotype of the expression strains ${ }^{15}$. Taken together, these results raise the interesting possibility that the SRTA protein acts mainly on naked DNA that enters the cell (i.e., transgenic DNA and possibly other types of invading DNA sequences), and inactivates them through the establishment of a repressive chromatin structure.

Genome-wide analysis of transposon mobility. A major function of silencing pathways is to control mobile genetic elements 37,38 . Our DNA gel blot analyses (Fig. 1f) had provided preliminary evidence for suppression of transposon mobility not being affected in our expression strains. Having obtained wholegenome sequencing data for the mutant strains UVM4 and UVM11 as well as the control strains Elow47 and cw15argenabled us to comprehensively assess the effect of the SRTA mutations on transposon activity in the expression strains (Supplementary Data 8). To this end, the DNA sequencing datasets were used to perform a genome-wide survey of nine different transposons in the four strains. In total, 656 transposons were found, and the vast majority of them (96\%) were in the same loci in all four strains (Table 2). The retrotransposon MRC1 was somewhat exceptional in that it showed multiple transposition events, which however, were not correlated to the presence of an SRTA mutation. Instead, the presence of multiple new insertions in all strains (including the control strains) 
a
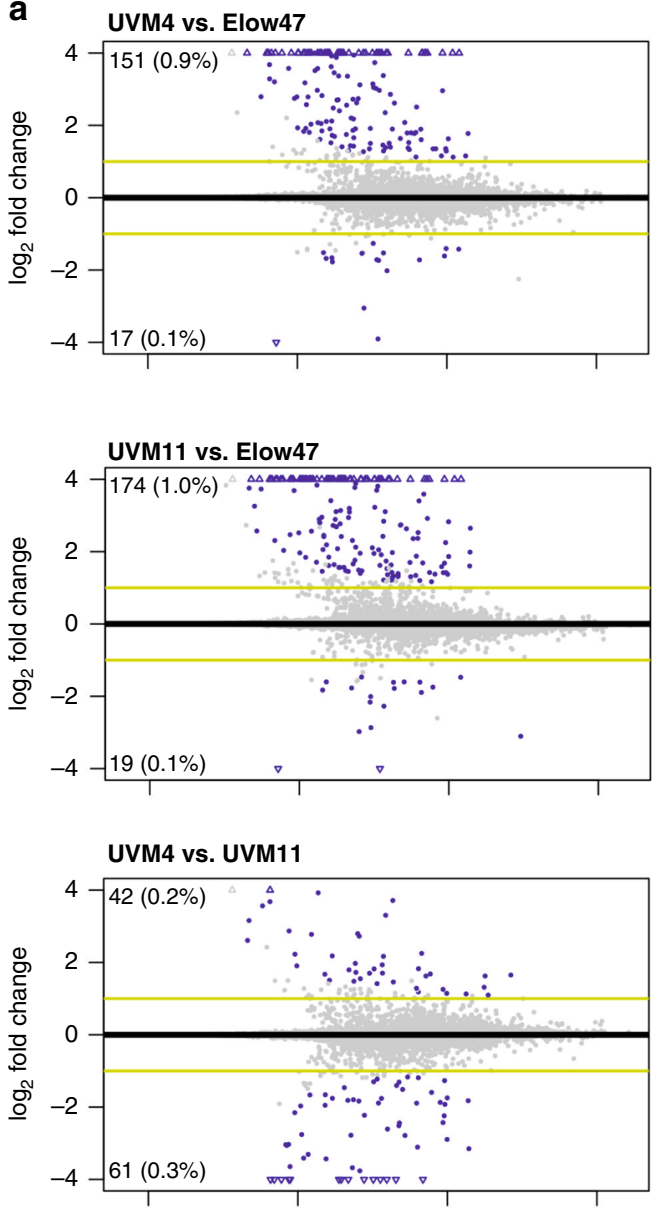

UVM11-C1 vs. UVM11

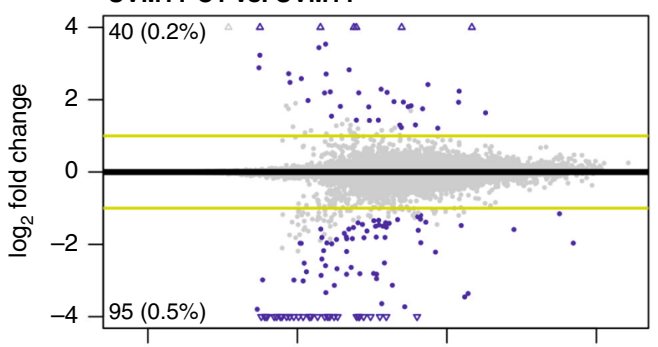

UVM11-C9 vs. UVM11

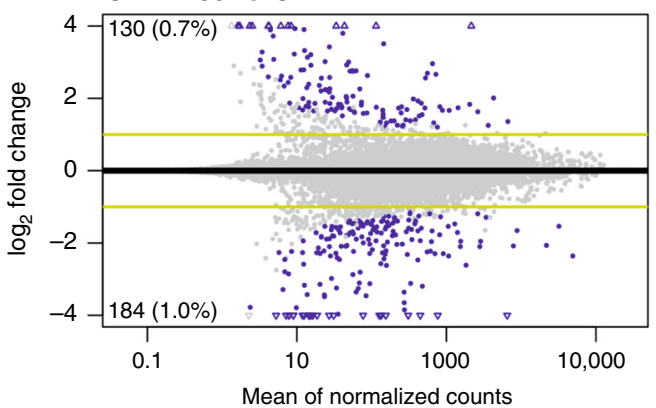

b

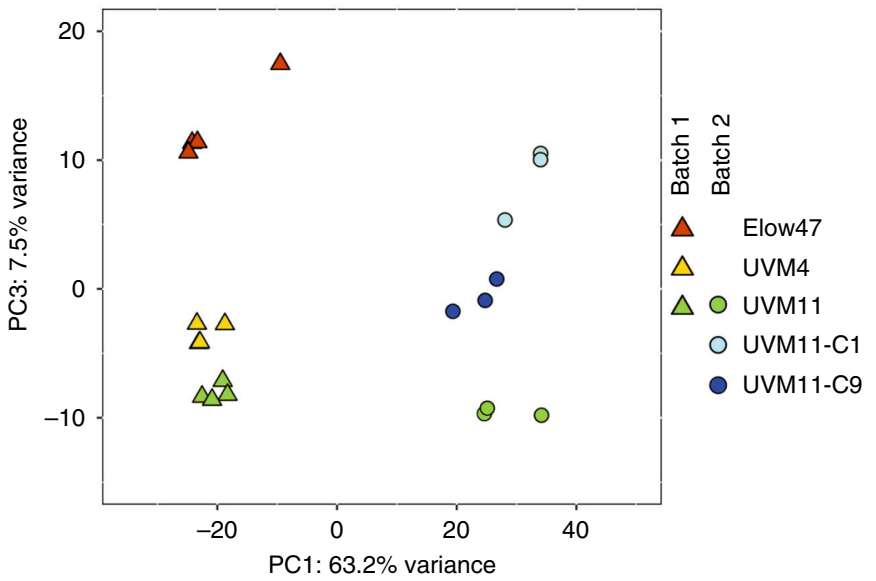

C

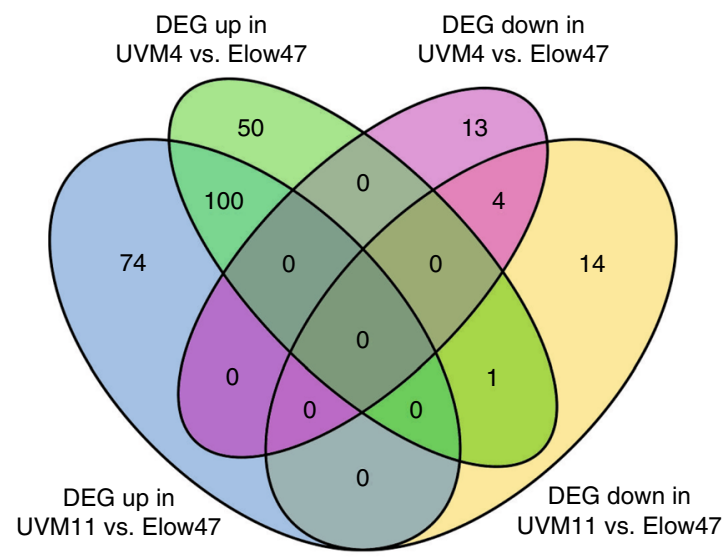

d

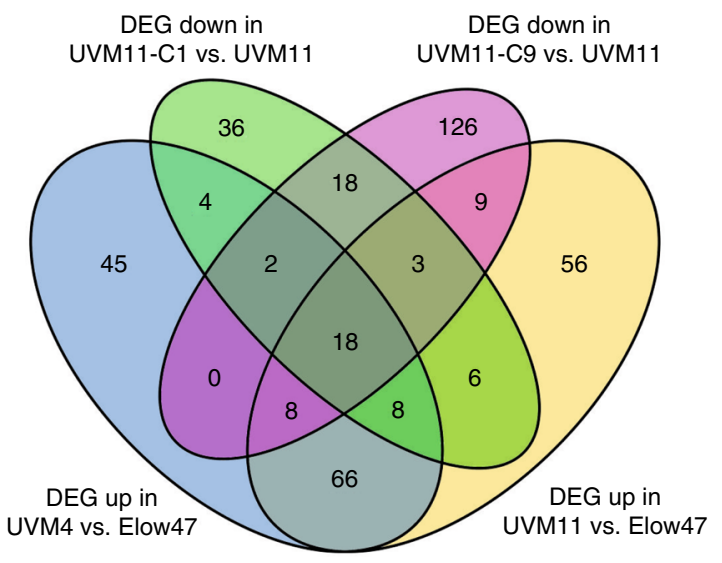

\section{Discussion}

In this study, we have identified the molecular basis of the strong transgene suppression in the reference alga Chlamydomonas. Performing mapping and genotyping by transformation, we identified the expression-conferring mutations in a highly conserved domain of a Sir2-type histone deacetylase (Cre10.g462200 
Fig. 6 Transcriptome analysis. srta mutant strains: UVM4, UVM11; the control strain: Elow47 (harboring the SRTA wild-type allele); and two complemented mutants: UVM11-C1 and UVM11-C9. a Visualization of differentially expressed genes (DEGs) by MA plot. RNAseq data were analyzed to identify DEGs in head-to-head comparisons between strains as follows: UVM4 (srta-2) and UVM11 (srta-1) were compared to Elow47 (SRTA) and with

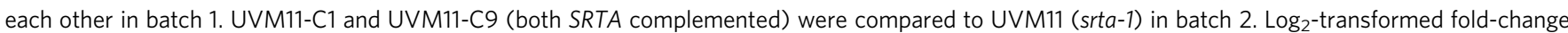
values are plotted for all genes. A gene was considered to be a DEG (highlighted in blue) if the absolute value of the $\log _{2}$ fold-change was $>1$ (indicated by an yellow line), and the Benjamini-Hochberg adjusted $p$-value was $<0.01$. The number of upregulated and downregulated DEGs is indicated in the upper left and lower left corners, respectively, in each panel. b Principal component analysis of RNAseq batch 1 consisted of strains Elow47, UVM4 (srta-2) and UVM11 (srta-1), with four biological replicates each. Batch 2 consisted of strains UVM11 (repeated from batch 1 to identify batch effects), UVM11-C1 and UVM11-C9, with three biological replicates each. A principal component analysis (PCA) plot was constructed from the 1000 genes with the highest variance across all samples. Each replicate is plotted according to the included legend. Note that, if the 1000 genes that are the most variable in this dataset are analyzed, only the third principal component (PC3) corresponds, to a limited extent, with the SRTA allele status, and PC3 only represents $7.5 \%$ of the variance. This represents a very small effect compared to the differences between batches, even though every effort was made to treat the samples the same. c Venn diagram showing the number of DEGs upregulated and downregulated in UVM11 and UVM4 relative to Elow47. Genes were identified as differentially expressed in comparisons between the strains if the fold change was $>2$, the mean FPKMs across all samples was $>1$, and the BenjaminiHochberg adjusted $p$-value was $<0.01$. d Venn diagram of overlap between DEGs as identified from RNAseq analyses of batch 1 and batch 2 . In order to identify patterns of expression that potentially correlate with SRTA allele status, a Venn diagram of DEGs was generated to identify the overlap between the following four comparisons: the number of upregulated DEGs in UVM11 versus Elow47, the number of upregulated DEGs in UVM4 versus Elow47, the number of downregulated DEGs in UVM11-C1 versus UVM11, and the number of downregulated DEGs in UVM11-C9 versus UVM11. The resulting genes and their expression estimates are presented in Supplementary Data 6 and 7. Source data are provided as a Source Data file.

Table 2 Presence of transposons in the wild-type strain cw15arg-, the wild type-like strain Elow47 and the srta mutant strains UVM4 and UVM11 as determined by whole-genome sequencing (cf. Supplementary Data 8).

\begin{tabular}{lrllllll} 
Transposon & Total number & $\begin{array}{l}\text { Present in all } \\
\text { strains }\end{array}$ & $\begin{array}{l}\text { In cw15arg- } \\
\text { only }\end{array}$ & $\begin{array}{l}\text { In Elow47 } \\
\text { only }\end{array}$ & $\begin{array}{l}\text { In UVM4 } \\
\text { only }\end{array}$ & $\begin{array}{l}\text { In UVM11 } \\
\text { only }\end{array}$ & $\begin{array}{l}\text { In Elow47 + UVM4 + } \\
\text { UVM11 }\end{array}$ \\
\hline MRC1 & 178 & 154 & 3 & 3 & 4 & 7 & 7 \\
Bill & 12 & 12 & 0 & 0 & 0 & 0 & 0 \\
Gulliver & 274 & 273 & 0 & 0 & 0 & 0 & 1 \\
Pioneer1 & 2 & 2 & 0 & 0 & 0 & 0 & 0 \\
REM1 & 12 & 12 & 0 & 0 & 0 & 0 & 0 \\
Trr1 & 8 & 8 & 0 & 0 & 0 & 0 & 0 \\
Tcr3 & 59 & 57 & 0 & 1 & 0 & 1 & 0 \\
TOC1 & 59 & 59 & 0 & 0 & 0 & 0 & 0 \\
TOC2 & 52 & 52 & 0 & 0 & 0 & 0 & 0 \\
\hline
\end{tabular}

encoding SRTA). Sirtuins represent a highly conserved family of $\mathrm{NAD}(+)$ dependent protein deacetylases with the yeast histone deacetylase Sir2 (silent information regulator) as the founding family member ${ }^{39}$. The algal expression strains UVM4 and UVM11 both carry non-synonymous point mutations in the SRTA gene, at different positions within the highly conserved Sir2 domain. Complementation experiments and characterization of an insertion mutant line confirmed the functional role of the $\mathrm{NAD}(+)$ dependent HDAC in epigenetic transgene suppression.

Knock-out of SIRT6, a representative of the same sirtuin clade (class IV; Fig. 3c; ref. ${ }^{26}$ ) and the closest homologue of SRTA in mammals, causes a severe phenotype in mice. This is unsurprising given that SIRT6 binds to the promoter region of thousands of genes in mice fibroblasts ${ }^{40}$. By contrast, the Chlamydomonas SRTA acts very differently from its mammalian SIRT6 homolog in that srta mutants have no discernable phenotype ${ }^{15}$. Moreover, the results of our comparative RNAseq analyses (Fig. 6) exclude a function of SRTA as a general transcriptional regulator of endogenous genes in the genome.

Instead, our results indicate that the effect of the loss of SRTA function is largely transgene-specific. Endogenous genes remain unaffected, as evidenced by our comparative transcriptomic analysis, suggesting that SRTA may act preferentially on DNA that newly enters the cell, is naked and not yet covered by chromatin proteins. Once the foreign gene is integrated into the genome and an active expression status has been established, it is much less susceptible to SRTA-mediated silencing than upon initial introduction into the cell. It should be noted, however, that the action of SRTA is not entirely limited to unintegrated naked DNA. In fact, the genetic screen that led to the isolation of the UVM4 and UVM11 mutants was based on the post-integration loss of transgene silencing ${ }^{15}$, suggesting that, at least under certain conditions, loss of SRTA function can also lead to activation of silent transgenes. The UV mutagenesis applied in the genetic screen could be one such condition in that it may have triggered DNA repair pathways that involve transient removal of histones.

An alternative explanation for the high transgene expression capacity of the srta mutants would be that the loss of SRTA predisposes the transgenes to insert into more favorable (euchromatic) genomic locations for expression. Once a transgene has been inserted into such a location, its continued expression may no longer depend on the presence of the srta mutation. In view of our findings that SRTA function is not involved in the control of endogenous gene expression (Fig. 6) and its loss does not lead to global changes in chromatin structure (Fig. 1; Supplementary Fig. 2), we consider this explanation less likely. Since the mutations identified in the two expression strains affect the catalytic domain of SRTA (and gene expression levels are unaffected), the phenotype is most likely associated with HDAC activity and the setting of histone marks on transgenic DNA entering the nucleus. Also, our ChIP data suggest that, in the expression strains, transgenes can also integrate into chromosomal regions that carry heterochromatic marks (Fig. 1e).

Genome resequencing and a genome-wide survey of the copy numbers and locations of transposable elements have allowed us to also exclude a role of SRTA in the control of mobile genetic 
elements. This, together with the unaltered sensitivity of the mutant strains to chemically induced DNA damage, the absence of significant effects of SRTA on the expression of endogenous genes in the genome and the lack of any detectable phenotype of srta mutants, suggests that SRTA plays no substantial role in the maintenance of genome stability or in nuclear gene regulation. Instead, all this evidence points to a transgene-specific action of SRTA in that it specifically silences foreign DNA after its entry into the cell, perhaps even prior to (or concurrently with) its integration into the genome. The inability of several pharmacological inhibitors of HDACs to phenocopy the expression phenotype of srta mutants may lend further support to this hypothesis. We currently cannot exclude the possibility that the primary recognition of the foreign DNA is mediated by an unknown protein factor that then recruits SRTA to assemble a repressive chromatin state. Nonetheless, even if additional factor (s) are involved in the molecular recognition process, SRTA appears to be the crucial factor that initiates silencing in a transgene-specific manner.

Invading nucleic acids are particularly prevalent in aquatic environments and, due to the absence of a protected germline, unicellular organisms are particularly susceptible to foreign nucleic acid sequences. We, therefore, speculate that SRTA silencing of foreign DNA may have evolved as a protective mechanism to prevent the expression of viruses and other potentially deleterious types of environmental DNA sequences that may constantly enter algal cells through horizontal DNA transfer ${ }^{4}{ }^{1}$. Interestingly, only relatively few lytic viruses are known in eukaryotic algae, and not a single virus is known that would infect Chlamydomonas ${ }^{42,43}$, possibly suggesting that algae have evolved unusually strong protective mechanisms against infectious nucleic acids.

In summary, we conclude from our data that foreign DNA that enters the cell is the preferred target of SRTA-mediated silencing. This suggests that SRTA may provide protection from potentially harmful types of environmental DNA. Further work will be needed to test, if naked DNA (that is not yet covered by chromatin proteins) is the only target of SRTA-mediated silencing, and if srta mutants have a selective disadvantage under conditions that are linked to the exposure to invading environmental DNA, including DNA viruses that infect other species of green algae.

Finally, the absence of any substantial deregulation of the transcriptome upon mutational inactivation of SRTA, the lack of evidence for genome destabilization by unleashed transposons, and the long-term stability of high-level transgene expression make the UVM strains a highly valuable tool for the efficient expression of foreign genes in Chlamydomonas.

\footnotetext{
Methods

Algal strains and cultivation conditions. Chlamydomonas reinhardtii strains Elow47, UVM4 and UVM11 were generated in a previous study ${ }^{15}$. Strain Elow47 is a co-transformant of the arginine auxotrophic strain CC-4350 (cw15, $m t+$, arg7; alias $c w 15$ arg-) that carries the CRY1-1 emetine resistance gene and the ARG7 gene providing arginine prototrophy. The expression strains UVM4 and UVM11 were obtained by UV-light-induced mutagenesis of strain Elow47, followed by selection for high transgene expression capacity ${ }^{15}$. Chlamydomonas strains CC1690 ( $m t+$; Sager 21gr), CC-124 ( $m t-$ ), and the polymorphic strain CC-2290 ( $m t-$, alias S1-D2) were obtained from the Chlamydomonas Stock Center (http:// chlamycollection.org/strains/). The insertion mutants srta-3 (LMJ.RY0402.148523, Cre10.g462200) and smm12 (LMJ.RY0402.153656, Cre03.g200350) and the corresponding wild-type strain CC-5325 are from the Chlamydomonas mutant library (CLiP, https://www.chlamylibrary.org/) and were provided by the Chlamydomonas Stock Center.

Cells were grown photomixotrophically in liquid Tris-acetate-phosphate (TAP) medium ${ }^{44,45}$ on a rotary shaker at $22-25^{\circ} \mathrm{C}$ or on agar-solidified TAP medium under continuous light $\left(50-100 \mu \mathrm{E} \mathrm{m} \mathrm{m}^{-2} \mathrm{~s}^{-1}\right.$, cool-white fluorescent lamps). Arginine $\left(100 \mu \mathrm{g} \mathrm{mL}^{-1}\right)$ was added to the medium as required. Cell numbers were
}

determined by counting of 100-fold diluted samples in a Z2 Coulter Counter (Beckman Coulter, Krefeld, Germany).

Cloning of SRTA, srta-2 and construction of transformation vectors. Cloning of the coding sequence, the promoter $/ 5^{\prime} \mathrm{UTR}$ and the $3^{\prime} \mathrm{UTR}$ sequence of SRTA (Cre10.g462200) was performed on the basis of the gene model provided by Phytozome v10 (http://www.phytozome.net/). The sequence comprising the reading frame of the mutated srta-2 allele (carrying the point mutation detected in strain UVM4) and additional 31 bp upstream was amplified with primers SRT3402fw (5'-TACTAGCTTACGAACAGCGCC-3') and SRT3402rvEcoRI (5' agaatTCACGCGTCATCGTCACC- $\left.3^{\prime}\right)$ using oligo(dT)-primed CDNA of strain UVM4 as a template and introducing an EcoRI site (underlined) at the $3^{\prime}$ end of the amplicon, followed by cloning into the $\mathrm{pCR}^{\mathrm{TM}} 2.1-\mathrm{TOPO}^{\circledR} \mathrm{TA}$ vector (Invitrogen, Karlsruhe, Germany). To clone the promoter of SRTA, the $5^{\prime}$ end of the gene was amplified with primers P-SRT-BamHI-fw (5'-aggatCCGCGC-

CATGTCCC- $3^{\prime}$, nucleotides introducing a BamHI site underlined) and NheI-SRTrv (5'-CGCTAGCTCCTTGACGCCC-3', NheI site underlined) using genomic DNA of strain Elow47 as a template. The $3^{\prime}$ UTR of SRTA was amplified from the same template using primers T-SRT-EcoRIfw (5'-agaattcAGGTGCTGTGTTGGGATG-3', nucleotides introducing an EcoRI site underlined) and T-SRT-XbaIrv (5'-TTCTAGACGTCGATGTATCCTGTGGTG-3', XbaI site underlined), and cloned into the $\mathrm{pCR}^{\mathrm{TM}} 2.1-\mathrm{TOPO}^{\circledR} \mathrm{TA}$ vector (Invitrogen). Subsequently, the srta2 coding sequence-containing TOPO vector was digested with BamHI and NheI and ligated to the similarly digested PCR product of the 5' end of the SRTA gene. The resulting pTOPO-P-uvm4 vector was digested with EcoRI and XbaI, and ligated to the insert of the similarly digested $\mathrm{pTOPO}$ vector that contained the SRTA 3'UTR sequence, resulting in plasmid pTOPO-P-uvm4-T.

Plasmid pJR80 was constructed by digesting plasmid pTOPO-P-uvm4-T with BamHI and XmaI, and replacing the $5^{\prime}$ end of the srta-2 sequence containing the point mutation with the similarly digested SRTA wild-type sequence. The SRTA sequence was amplified by an overlap extension PCR in which amplicon 1 (generated with primers P-SRT-BamHI-fw and SRT-E2wtfw [5'GTGTTCACGGGCGCCGGCATCTCCACCGCCTGCGGCATCC-3', nucleotide reverting the point mutation in srta-2 in bold] and using pTopo-P-uvm4-T as template) and amplicon 2 (generated with primers SRT-E2wt-rv [5'GGATGCCGCAGGCGGTGGAGATGCCGGCGCCCGTGAACAC- ${ }^{\prime}$, nucleotide reverting the point mutation in srta-2 in bold] and SRTXmaI-rv [5'ACCCCGGGGCCACTGTCAG- $3^{\prime}$ ] using pTOPO-P-uvm4-T as template) were used as templates and amplified with primers P-SRT-BamHI-fw and SRTXmaI-rv

Chlamydomonas transformation vector pJR85 was obtained by digestion of plasmid pJR43 (a derivative of the $A P H 7$ "-containing pHyg3 vector; ref. ${ }^{46}$ ) with $\mathrm{XbaI}$ and HindIII followed by ligation to the $2988 \mathrm{bp}$, gel-eluted XbaI/HindIII fragment of pTOPO-P-uvm4-T that contained the srta-2 sequence including the SRTA promoter and terminator sequences. Plasmid pJR43 was generated by ligating the purified KpnI/EcoRV hygromycin cassette $\left(P_{T U B 2}:: A P H 7^{\prime \prime}\right)$ from $\mathrm{pHyg} 3$ into the similarly digested $\mathrm{pBC} \mathrm{KS}(+)$ vector.

Chlamydomonas transformation vector pJR81 carrying the SRTA sequence under the control of the SRTA promoter and terminator sequences was obtained by digesting plasmid pJR85 with ClaI and SmaI, and ligating the eluted vector with the gel-eluted insert from the similarly digested plasmid pJR80. Plasmid pJR88 was obtained by MluI digestion of plasmid pJR81 followed by dephosphorylation using Fast Alkaline Phosphatase (Thermo Fisher Scientific, Darmstadt, Germany) and ligation to the annealed double-stranded oligonucleotide sequence that has MluIcompatible single-stranded overhangs at the $5^{\prime}$ and $3^{\prime}$ ends, and encodes the FLAG sequence. The dsDNA fragment was generated by annealing sense (SRT-FLAGfw, $5^{\prime}$ cgcgGACTACAAGGACGACGACGACAAGTAA $3^{\prime}$ ) and antisense oligonucleotide (SRT-FLAGrv, 5' cgcgTTACTTGTCGTCGTCGTCCTTGTAGTC $3^{\prime}$ ) followed by phosphorylation with T4 polynucleotide kinase (New England Biolabs, Frankfurt/M., Germany).

In addition to the Venus YFP-containing vector pJR39 (ref. ${ }^{15}$ ) and vector pRMB12 containing the fully codon-optimized CrYFP ${ }^{14}$ that have the APHVIII gene as a selectable marker, another Venus YFP-containing plasmid with a different selectable marker gene was constructed (pJR91 carrying the APH7" gene). pJR91 was obtained by digestion of pJR43 and isolation of the APH7"-containing EcoRV/KpnI restriction fragment, followed by ligation to vector pJR39 cut with XhoI followed by treatment with the Klenow fragment of DNA polymerase I (New England Biolabs, Frankfurt/M., Germany) and digestion with KpnI.

Transformation of Chlamydomonas reinhardtii. Nuclear transformation of cell wall-deficient Chlamydomonas strains was performed using the glass bead-assisted $\operatorname{method}^{47,48}$. Transformations of cell wall-containing strains were done by electroporation using the Eppendorf 2510 electroporator or the Biorad Gene Pulser Xcell ${ }^{\mathrm{TM}}$ electroporation system. All transformation vectors were linearized with a restriction enzyme (RE) that has a unique recognition site within the vector backbone. One microgram of linearized plasmid was used for the transformation of $1 \times 10^{8}$ cells harvested from a culture grown until the early to mid-exponential phase. For electroporation, pelleted cells were dissolved in sterile water with $40 \mathrm{mM}$ sucrose in a volume of $500 \mu \mathrm{L}$ and transferred to a pre-cooled electroporation cuvette ( $4 \mathrm{~mm}$ gap; Biorad, Germany). Cells were incubated with the linearized plasmid DNA for 5-20 min on ice before an electric pulse of $1200 \mathrm{~V}$ was applied. 
After electroshock, cuvettes were immediately cooled on ice and cells were transferred into $11 \mathrm{~mL}$ TAP medium with $40 \mathrm{mM}$ sucrose for recovery under nonselective conditions for $\sim 4 \mathrm{~h}$. The cells were then pelleted and spread onto plates with selective medium. Supplementary Table 9 shows an overview of all transformation experiments conducted in this study, including information about the $\mathrm{RE}$ used for linearization of the vectors and the conditions used for the selection of transformants.

DNA isolation and standard PCRs. Extraction of genomic DNA was performed according to published protocols ${ }^{14}$ using $2 \mathrm{~mL}$ of algal cultures grown in liquid medium to late-exponential phase or a similar number of cells grown on solid medium. The cell pellet was resuspended in $200 \mu$ l extraction buffer ( $2 \%$ cetyltrimethylammonium bromide, $100 \mathrm{mM}$ Tris- $\mathrm{HCl} \mathrm{pH}$ 8.0, $20 \mathrm{mM}$ EDTA pH 8.0, $1.4 \mathrm{M} \mathrm{NaCl}$, and $2 \%$ freshly added $\beta$-mercaptoethanol) by vortexing, followed by the addition of $200 \mu \mathrm{L}$ chloroform/isoamyl alcohol $(24: 1)$ and incubation at $65^{\circ} \mathrm{C}$ for $20 \mathrm{~min}$ under shaking $(1400 \mathrm{rpm})$. After centrifugation at $10^{\circ} \mathrm{C}$ for $10 \mathrm{~min}$ at maximum speed, the DNA was precipitated by adding 0.7 volume of isopropanol to the supernatant. The pellet was washed with $70 \%(\mathrm{v} / \mathrm{v})$ ethanol and the air-dried pellet was resuspended in $50 \mu \mathrm{L}$ water supplemented with $1 \mu \mathrm{L}$ RNase $\mathrm{A}\left(10 \mathrm{mg} \mathrm{mL}^{-1}\right)$, and incubated for $15 \mathrm{~min}$ at $37^{\circ} \mathrm{C}$.

Alternatively, crude DNA extracts were isolated for PCR genotyping according to the instructions given by the Chlamydomonas mutant library (CLiP, https:// www.chlamylibrary.org/) with minor modifications. In brief, Chlamydomonas cells collected from plates were resuspended in $50 \mu \mathrm{L}$ of $10 \mathrm{mM}$ EDTA (pH 8.0) by vortexing for $10 \mathrm{~s}$, followed by incubation at $100^{\circ} \mathrm{C}$ for $10 \mathrm{~min}$ in a thermocycler. After cooling for $1 \mathrm{~min}$ at $4{ }^{\circ} \mathrm{C}$, the samples were again vortexed for $10 \mathrm{~s}$ and centrifuged for $1 \mathrm{~min}$ at $2000 \times g$. The DNA-containing supernatant was then transferred to fresh tubes and used for PCR analysis. Polymerase chain reactions (PCRs) were performed according to standard protocols $\left(1 \mathrm{~min}\right.$ at $98^{\circ} \mathrm{C}, 90 \mathrm{~s}$ at $58-66^{\circ} \mathrm{C}, 90$ s at $72{ }^{\circ} \mathrm{C} ; 36-40$ cycles) using GoTaq ${ }^{\oplus}$ DNA polymerase (Promega, Mannheim, Germany). Primer sequences are listed in Supplementary Table 10.

Southern blot analysis to determine transposon copy numbers. Samples of 40 or $60 \mu \mathrm{g}$ total DNA were digested with the RE HincII or HindIII to analyze the copy number of the retrotransposon-like element $T O C 1$ and the DNA transposon Gulliver, respectively. Digested total DNA samples were separated in $1 \%$ agarose gels and transferred onto Hybond ${ }^{\mathrm{TM}} \mathrm{N}^{+}$nylon membranes (GE Healthcare) by capillary blotting. The TOC1 probe was amplified by PCR (primers TOCpos $2075^{\prime}-$ GGGACGGTGACCTCAGTGTGTCGC- $3^{\prime}$ and TOCpos $4745^{\prime}$-CCGCTCTGCT CCGATTTGCTCCCG-3'), cloned into the $\mathrm{pCR}^{\mathrm{TM}} 2.1-\mathrm{TOPO}^{\circledR} \mathrm{TA}$ vector (Invitrogen), digested with EcoRI and purified by agarose gel electrophoresis followed by labeling with ${ }^{[\alpha-32 \mathrm{P}]}$ dCTP by random priming (Megaprime ${ }^{\mathrm{TM}} \mathrm{DNA}$ labeling system, GE Healthcare, Freiburg, Germany). The Gulliver probe was amplified by PCR (GULLIVfw2 $5^{\prime}$-CGCAGGCAAGCGAAGGCTCA-3' and LTRrev 5'- AAGT GCCTTTGTGGAAGCCATGT-3') and purified by gel electrophoresis prior to labeling. Hybridizations took place at $65^{\circ} \mathrm{C}$ using standard protocols.

Protein isolation and immunoblot analysis. Isolation of total soluble protein extracts, separation of proteins via SDS page and immunoblot analysis were performed according to published protocols ${ }^{15}$ with minor modifications. In brief, algal cell pellets were resuspended in $400 \mu \mathrm{L}$ lysis buffer [ $50 \mathrm{mM} \mathrm{HEPES/KOH} \mathrm{pH} \mathrm{7.5,}$ $10 \mathrm{mM} \mathrm{KAc}, 5 \mathrm{mM}$ MgAc, $1 \mathrm{mM}$ EDTA, $1 \mathrm{mM}$ DTT, $1 \times$ protease inhibitor cocktail cOmplete (Roche)], followed by disruption of cells by sonication (Sonifier ${ }^{\circ}, \mathrm{W}-250$ D) at $10 \%$ amplitude for $15 \mathrm{~s}$ while keeping samples on ice. After centrifugation at $4{ }^{\circ} \mathrm{C}$ for $10 \mathrm{~min}(10,000-12,000 \times g)$, the supernatant was transferred to a fresh tube and protein concentration was determined using the Bradford assay (Roti ${ }^{\circ}$ Quant, Roth, Karlsruhe). Heat denaturation of the protein samples in $1 \times$ sample buffer took place at $75^{\circ} \mathrm{C}$ for $7 \mathrm{~min}$ or at $95^{\circ} \mathrm{C}$ for $5 \mathrm{~min}$. Protein samples were separated in denaturing 15\% SDS-PAA gels and transferred onto PVDF (polyvinylidene difluoride) membranes (Hybond ${ }^{\mathrm{TM}}$; GE Healthcare, UK) using standard Trisglycine transfer buffer $(25 \mathrm{mM}$ Tris/HCl, $192 \mathrm{mM}$ glycine, $\mathrm{pH}$ 8.3). After electrophoretic transfer, membranes were treated with blocking buffer composed of $0.5 \%$ BSA and $0.5 \%$ milk powder in $1 \times$ TBS-T.

For immunobiochemical protein detection of global histone $\mathrm{H} 3$ and specific histone modifications the following primary antibodies were used in different dilutions in TBS-T: 1:50,000 dilution of anti-H3 (ab1791; Abcam, Cambridge, UK), 1:10,000 dilution of anti-H3ac (03-599; Merck, Darmstadt, Germany), 1:1000 of anti-H4ac (K5, 8, 12, 16; AHP418; Biorad), 1:5000 of anti-H3K4me1 (ab8895; Abcam) and 1:1000 of anti-H3K4me2 (07-030; Merck). Detection of YFP was performed with a 1:1000 dilution of monoclonal anti-GFP primary antibody (632381; Clontech, Mountain View, CA). Gels were loaded based on equal amounts of total soluble protein. Immunochemical detection was performed with the ECL Plus ${ }^{\mathrm{TM}}$ detection system (GE Healthcare) and a goat anti-rabbit secondary antibody (170-6515; Biorad, Munich, Germany) or, for YFP detection, a goat antimouse HRP-conjugated secondary antibody (AS111772; Agrisera, Vännäs, Sweden), using a 1:10,000 dilution.

Chromatin immunoprecipitation. For each ChIP experiment, at least 1200 YFP transformants (with vector pJR39) of each strain (Elow47, UVM4, and UVM11) were pooled and a total of $10^{8}$ algal cells were harvested by a 2-min centrifugation at $4{ }^{\circ} \mathrm{C}$ and $3220 \times g$. The chromatin was cross-linked by resuspending cells in 10 $\mathrm{mL}$ of freshly prepared cross-linking buffer (20 mM HEPES-KOH, $\mathrm{pH} 7.6,80 \mathrm{mM}$ $\mathrm{KCl}, 0.35 \%$ formaldehyde), and incubated for $10 \mathrm{~min}$ at $24^{\circ} \mathrm{C}$. Subsequently, crosslinking was quenched by the addition of glycine to a final concentration of 125 $\mathrm{mM}$, and continued incubation for $5 \mathrm{~min}$ at $24^{\circ} \mathrm{C}$. Cells were then pelleted by centrifugation for $2 \mathrm{~min}$ at $4{ }^{\circ} \mathrm{C}$ and $3220 \times g$, followed by cell lysis by addition of $400 \mu \mathrm{L}$ lysis buffer (1\% SDS, $10 \mathrm{mM}$ EDTA, $50 \mathrm{mM}$ Tris- $\mathrm{HCl}, \mathrm{pH}$ 8.0, $0.25 \times$ protease inhibitor cocktail [Roche]) and sonication on ice using a BANDELIN Sonopuls HD 2070 sonicator with sonication tip MS 73 (55\% output control and $60 \%$ duty cycle) to obtain an average DNA fragment size of $\sim 200 \mathrm{bp}$. ChIP was performed according to published procedures ${ }^{49}$. In brief, culture aliquots corresponding to $\sim 2 \times 10^{7}$ algal cells were diluted 1:10 with ChIP buffer (1.1\% Triton X100, $1.2 \mathrm{mM}$ EDTA, $167 \mathrm{mM} \mathrm{NaCl}, 16.7 \mathrm{mM}$ Tris- $\mathrm{HCl}, \mathrm{pH}$ 8), and supplemented with BSA and sonicated $\lambda$-DNA at final concentrations of 100 and $1 \mu \mathrm{gL}^{-1}$ respectively. Antibodies specifically recognizing the following epitopes were used: histone H3 (5 $\mu \mathrm{L}$; Abcam ab1791); diacetyl H3K9 and H3K14 (10 $\mu \mathrm{L}$; Upstate 06599); tetra-acetyl $\mathrm{H} 4 \mathrm{~K} 5, \mathrm{H} 4 \mathrm{~K} 8, \mathrm{H} 4 \mathrm{~K} 12$, and $\mathrm{H} 4 \mathrm{~K} 16$ (10 $\mathrm{LL}$; Upstate 06-866); monomethylated H3K9 (5 $\mu \mathrm{L}$; ab9045; Abcam). Antibody-protein-DNA complexes were incubated for $1 \mathrm{~h}$ at $4{ }^{\circ} \mathrm{C}$. Subsequently, $6 \mathrm{mg}$ preswollen protein A Sepharose beads (Sigma-Aldrich) were added, followed by a $2 \mathrm{~h}$ incubation at $4{ }^{\circ} \mathrm{C}$. Sepharose beads were pelleted by centrifugation at $4{ }^{\circ} \mathrm{C}$ for $30 \mathrm{~s}$ at $12,000 \times g$, washed with washing buffer $1(0.1 \%$ SDS, $1 \%$ Triton X-100, 2 mM EDTA, pH 8) containing 150 $\mathrm{mM} \mathrm{NaCl}$, followed by additional washes with washing buffer 1 containing 500 $\mathrm{mM} \mathrm{NaCl}$ and washing buffer $2(250 \mathrm{mM} \mathrm{LiCl}, 1 \%$ Nonidet P- $40,1 \% \mathrm{Na}$-deoxycholate, $1 \mathrm{mM}$ EDTA, $10 \mathrm{mM}$ Tris-HCl, $\mathrm{pH}$ 8), and two washes with TE ( $1 \mathrm{mM}$ EDTA and $10 \mathrm{mM}$ Tris- $\mathrm{HCl}, \mathrm{pH}$ 8). Protein-DNA complexes were then eluted by incubation for $30 \mathrm{~min}$ at $65^{\circ} \mathrm{C}$ in elution buffer ( $1 \% \mathrm{SDS}, 0.1 \mathrm{M} \mathrm{NaHCO}_{3}$ ). Cross-links were reverted by overnight incubation at $65^{\circ} \mathrm{C}$ after the addition of $\mathrm{NaCl}$ to a final concentration of $0.5 \mathrm{M}$. Proteins were then digested by addition of $3.5 \mu \mathrm{g} \mathrm{mL}-1$ proteinase $\mathrm{K}, 8 \mathrm{mM}$ EDTA, and $32 \mathrm{mM}$ Tris- $\mathrm{HCl}$, $\mathrm{pH}$ 8.0, followed by incubation for $1 \mathrm{~h}$ at $55^{\circ} \mathrm{C}$. The solution was subsequently extracted once with $\mathrm{phenol/chloroform/isoamyl} \mathrm{alcohol} \mathrm{(25:24:1)} \mathrm{and} \mathrm{once} \mathrm{with} \mathrm{chloroform/isoamyl}$ alcohol (24:1), and the DNA was precipitated by incubation with 2 volumes of ethanol after addition of $0.3 \mathrm{M} \mathrm{Na}$-acetate, $\mathrm{pH} 5.2$, and $10 \mu \mathrm{g} \mathrm{mL}^{-1}$ glycogen for $3 \mathrm{~h}$ at $-20^{\circ} \mathrm{C}$. Precipitated DNA was pelleted by a 20 min centrifugation at $4{ }^{\circ} \mathrm{C}$ and $16,000 \times g$, washed with $70 \%$ ethanol, air-dried, and resuspended in TE buffer Aliquots representing $2.5 \%$ of the precipitated DNA were used as templates for qPCR. Signals for individual gene regions were normalized to $10 \%$ input DNA and then to the corresponding signal derived from the CYC6 promoter $(\mathrm{H} 3, \mathrm{AcH} 3$, $\mathrm{AcH} 4$ ) or a TFR (TFR; H3K9me1). qPCR assays were performed using the StepOnePlus RT-PCR system (Applied Biosystems) and the Maxima SYBR Green kit (Fermentas). Each amplification reaction contained the vendor's master mix, $200 \mathrm{nM}$ of each primer and ChIPed DNA corresponding to $2.5 \%$ of the total eluate. The reaction conditions were: $95^{\circ} \mathrm{C}$ for $10 \mathrm{~min}$, followed by cycles of $95^{\circ} \mathrm{C}$ for $15 \mathrm{~s}$ and $65^{\circ} \mathrm{C}$ for $60 \mathrm{~s}$, up to a total of 40 cycles. Primers used for qPCR are listed in Supplementary Table 10 .

Crossings, mapping, and PCR marker analysis. Crosses were performed according to standard procedure ${ }^{44}$. Prior to the mapping of the mutated locus in the expression strains, the cell wall-deficient strain UVM11 $(m t+)$ that displayed poor mating capability was back-crossed to the mt- wild-type strain CC-124 (Fig. 2a) to restore flagella as a prerequisite for efficient mating. From this cross, the offspring strain UVM11-CW $(m t+)$ was isolated that showed strong YFP expression as confirmed by microscopy analysis of several independent YFP transformants. Strain UVM11-CW $(m t+)$ was then crossed to mapping strain CC2290 ( $m t-$; alias S1-D2) and the progeny was screened for its transgene expression capacity by transforming every single segregant with the YFP reporter gene followed by YFP fluorescence analysis. Progeny with high YFP expression was used as the mapping population and selected for PCR marker analysis using the Chlamydomonas mapping kit available from the Chlamydomonas resource center (http://www.chlamycollection.org/products/mapping-kits/). Newly developed markers for fine mapping are listed in Supplementary Table 10. Segregants were scored as having strong YFP expression capacity (i.e., showing the expression phenotype) if at least $30 \%$ of the colonies analyzed showed clearly visible YFP fluorescence. On average, 20 independent transformants were analyzed.

Zeocin sensitivity assay. To analyze the sensitivity of the mutant strains UVM4 and UVM11 to DNA-damaging agents, zeocin (Invivogen) sensitivity assays were performed. Cells of a late-exponential culture were diluted to three different cell concentrations $\left(1 \times 10^{7}, 1 \times 10^{6}\right.$, and $2 \times 10^{5}$ cells $\left.\mathrm{mL}^{-1}\right)$ and from each dilution, aliquots of $8 \mu \mathrm{l}$ were dropped onto TAP agar plates containing different concentrations of zeocin $\left(0,1,2\right.$, and $\left.4 \mu \mathrm{g} \mathrm{mL}^{-1}\right)$. Photos of the drop tests were taken after ten days.

Analysis of GFP and YFP fluorescence. Fluorescence of YFP was detected in living cells with a fluorescence stereomicroscope (Axioskop 2; Zeiss, Göttingen, Germany) equipped with appropriate filters for YFP (excitation BP 450-490 nm, FT 510, emission BP 515-565 nm, or excitation BP $470 / 20 \mathrm{~nm}$, FT 493, emission 
BP 505-565 nm) and chlorophyll fluorescence (excitation BP 450-490 nm, FT 510, long pass filter $520 \mathrm{~nm}$ ). Alternatively, the detection was done with a confocal laserscanning microscope (TCS SP5; Leica, Wetzlar; Germany) using an argon laser for excitation (at $514 \mathrm{~nm}$ for YFP), a $510-535 \mathrm{~nm}$ filter for detection of YFP fluorescence and a 630-720 nm filter for detection of chlorophyll fluorescence. YFP fluorescence intensity was measured with the CLARIOstar ${ }^{\circledR}$ microplate reader (BMG LABTECH GmbH, Ortenberg, Germany) in transparent 96-well plates with cultures of $100 \mu \mathrm{l}$ Chlamydomonas cells per well (grown to mid-exponential phase) using bottom optics and excitation (508 $\mathrm{nm}$, bandwidth $15 \mathrm{~nm}$ ) and emission $(551 \mathrm{~nm}$, bandwidth $20 \mathrm{~nm}$ ) settings suitable for YFP detection (dichroic 528.2). Scanning of Chlamydomonas clones grown on TAP agar plates for YFP fluorescence was conducted with the Typhoon scanner (GE Healthcare) applying the 526 SP Fluorescein filter.

RNA isolation and cDNA synthesis. Total RNA was isolated from algal cultures grown to late-exponential phase with the Direct-Zol ${ }^{\mathrm{TM}} \mathrm{RNA}$ MiniPrep kit (Zymo Research, Freiburg, Germany) followed by an additional DNase treatment using TURBO DNase (Thermo Fisher Scientific) according to the manufacturer's instructions. RNA yield and quality were assessed by determining the $260 \mathrm{~nm} /$ $280 \mathrm{~nm}$ absorbance ratio using a Nanodrop instrument (Thermo Fisher Scientific).

Unless otherwise stated, first-strand cDNA was synthesized from total RNA $(1.7-4 \mu \mathrm{g})$ using the RT primer mix (Qiagen) and Superscript III reverse transcriptase (Invitrogen) following the manufacturer's protocol. Subsequently, the RNA was removed by RNase H (Invitrogen) treatment. The absence of contaminating genomic DNA was confirmed by the inclusion of control samples that had not undergone reverse transcription. In order to determine the transcript ratio of the srta-1 mutant allele and the SRTA wild-type allele in the complemented lines of strain UVM11, the SRTA-specific primer SRT3402r2 (5'- GCTCGCCGG TCCTTGG- $3^{\prime}$ ) was used to prime the reverse transcription reactions. The sequence containing the srta-1 point mutation was amplified with primers SRTE5f (5'-AGCT GGTGGACAACATCCTG-3') and SRT8r (5'-GCGTCTTCTGCAGGTTCAC-3').

Whole-genome resequencing. G'enomic sequencing was performed on strains Elow47, UVM4, UVM11, and the wild-type cw15arg- according to published protocols ${ }^{50}$. In brief, $1 \mu \mathrm{g}$ of genomic DNA was used to prepare sequencing libraries using the TruSeq DNA sample preparation kit, version 1 (Illumina) following the low-throughput protocol. The resulting libraries were sequenced with paired-end $100+100$ nt reads on a HiSeq2000 sequencer (Illumina). Raw sequencing reads were aligned to version 5 of the Chlamydomonas reference sequence from Phytozome (http://phytozome.net) with BWA mem, version 0.7.5a-r405 (ref. ${ }^{51}$ ), using default parameters. Duplicate read pairs were removed using Picard MarkDuplicates, version 1.85(1345) (http:// broadinstitute.github.io/picard) with default parameters. Variants were identified with the Genome Analysis Toolkit (GATK), version 2.6-5-gba531bd. Loci that were identified as a reference in Elow47, but variants in either UVM4, UVM11 or both were selected for further analysis. The functional impact of each variant was predicted using SnpEff version $4.3 \mathrm{r}$ (ref. ${ }^{52}$ ). This produced a table of variants in VCF format, which was manually curated to identify the genes impacted by SNVs and InDels in either UVM4, UVM11, or both.

The sequencing reads are available from the NCBI Sequence Read Archive under accession numbers SRR1797981 (cw15arg-), SRR6872092 (Elow47), SRR6872091 (UVM4), and SRR6872090 (UVM11).

Transposon analysis. Paired-end $(100+100 \mathrm{nt})$ DNAseq was performed on strains cw15arg- (alias CC-4350), Elow47, UVM4, and UVM11 according to published protocols $s^{50}$. The resulting reads were mapped with bwa mem (v0.7.17r1188) using default parameters to the sequences of the following C. reinhardtii transposons from NCBI: Bill (DQ446204.1), Gulliver (AF019750.1 and AF019751.1), MRC1 (DQ446210.1), Pioneer1 (U19367.1), REM1 (AY227352.1), Tcr1 (DQ446205.1), Tcr3 (Y14652.1 and Y14653.1), TOC1 (X56231.1), and TOC2 (X84663.1). The resulting alignment files were filtered to isolate half-mapped reads (i.e., those with one end mapped and one end unmapped) with samtools (v 1.9-58gbd1a409). The remaining subset of half-mapped reads was then converted back to fastq format with samtools, and mapped to the C. reinhardtii genome assembly (v5; phytozome.net) with bwa mem, as before. The resulting alignment files were merged with samtools, and used to produce a bed file of total coverage with bedtools (v2.29.2) genomecov. Coverage data were used to identify the coordinates of transposons in these four strains as regions with at least 10 half-mapped reads. Next, the number of half-mapped reads from each strain was quantified with bedtools to determine the coverage for each transposon. These counts were combined into a single data table for further analysis in R. Each transposon at each locus in each strain was scored as present if $\geq 10$ reads mapped to it. To identify loci with transposons that differ between the four strains, the ratio of counts for the strain with the most counts versus the fewest counts was calculated for each locus. Those loci with a ratio $\geq 10$ were validated by manual curation in IGV version 2.8.10 (https://igv.org/).

RNAseq and data analysis. For RNAseq analysis, algal strains were grown in liquid TAP medium with the revised nutrient supplementation ${ }^{45}$ under continuous light $\left(90 \mu \mathrm{E} \mathrm{m}^{-2} \mathrm{~s}^{-1}\right)$. Once cells reached a concentration of $5-6 \times 10^{6}$ cells $\mathrm{mL}^{-1}$, a total of $5 \times 10^{7}$ cells were collected by centrifugation at $1424 \times g$ for $4 \mathrm{~min}$ at $4^{\circ} \mathrm{C}$. Total RNA was extracted with the TRIzol reagent as previously described ${ }^{20}$ with the following modifications. RNA was treated with DNase (Zymo Research), followed by a cleaning and concentration step with the RNA Clean \& Concentrator-5 kit (Zymo Research). RNA quality control and library preparation were performed by the Functional Genomics Laboratory (FGL), California Institute for Quantitative Biosciences (QB3), Berkeley. Samples were evaluated for quality on a 2100 expert Plant RNA Pico microfluidic chip on a Bioanalyzer 2100 (Agilent). Enrichment of poly(A) mRNA and library preparation was performed using the KAPA mRNA HyperPrep kit (Roche). Sequencing of the samples was performed on an Illumina HiSeq4000 by the Vincent J. Coates Genomics Sequencing Laboratory at UC Berkeley.

The sequenced reads were aligned to the $C$. reinhardtii reference genome (v.5) available from Phytozome (http://phytozome.net) by means of RNA-STAR (v2.4.0j). Default settings were used except for --alignIntronMax 3000. Aligned reads were then assigned to C. reinhardtii gene models (v.5.5) with the featureCounts program of the Rsubread package (v1.12.6) within the R statistical computing platform (v4.0.1). The resulting counts per gene were then used to identify DEGs and to plot a principal component analysis with the DESeq2 package (v1.18.1; https://bioconductor.org/packages/release/bioc/html/DESeq2.html) in R. Principal component analysis was performed in $\mathrm{R}$ with the prcomp function on the regularized log-transformed counts for the 1000 genes with the greatest variance, and plotted with ggplot2 (v3.3.2). MA plots were generated with the plotMA function in DESeq2 with alpha $=0.01$ and lfcThreshold $=1 . \log _{2}$ fold-change shrinkage was calculated with the apeglm method ${ }^{53}$ in R. A Venn diagram of DEGs was plotted with the VennDiagram package (v1.6.20) in R. Aligned reads were used to calculate normalized transcript abundances in terms of fragments per kbp of gene per million reads (FPKMs) with cuffdiff (v2.2.1). The density of FPKMs for the transcriptome of each strain was plotted with cummeRbund (v2.24.0) in R.

The RNAseq data, including raw reads and FPKM expression tables, were deposited in the NCBI Gene Expression Omnibus (GEO) database under accession GSE128981.

Protein homology and phylogenetic analyses. Gene and protein BLAST analyses were performed using the Phytozome web page (Phytozome v10, v11 or v12.1; http://www.phytozome.net/) and the NCBI database. The Saccharomyces genome database (http://www.yeastgenome.org/) and the HGNC (http://www.genenames. org/cgi-bin/genefamilies/set/937) database were used to extract all annotated histone deacetylases from yeast and humans, respectively. Sequences annotated as histone deacetylases in Arabidopsis and Chlamydomonas were identified in the Phytozome database v11 and v12.1. The Arabidopsis set was identical to that reported in a previous study ${ }^{24}$. Amino acid sequences of sirtuin family members from other organisms were obtained from NCBI (https://www.ncbi.nlm.nih.gov/)

For phylogenetic analysis of HDAC sequences, multiple sequence alignments were performed with ClustalW ${ }^{54}$ and neighbor-joining phylogenetic analysis was conducted with the MEGAX software (Molecular Evolutionary Genetics Analysis, https://www.megasoftware.net/mega4/). For phylogenetic tree construction, sirtuin sequences from model organisms representing different taxonomic groups were used: Chlamydomonas reinhardtii (CrSRTA-C; Cre10.g462200.t1.2, Cre12.g524650 t1.2, Cre05.g234663.t1.1), Saccharomyces cerevisiae (ScSir2p, ScHst1-4; YDL042C, YOL068C, YPL015C, YOR025W, YDR191W), Drosophila melanogaster (DmSirt1, 2, 4, 6, and 7; NP_477351.1, NP_001287422.1, NP_572241.2, NP_649990.2, NP 651664.2), Danio rerio (DrSIRT1-7; XP 005173091.1, NP 955890.1, NP_001073643.1, XP_005166125.1, XP_005170200.1, XP_021335349.1, XP_001336438.3), Homo sapiens (HsSIRT1-7; NP 036370.2, NP_036369.2, NP_036371.1, NP_036372.1, NP_036373.1, NP_057623.2, NP_057622.1), Arabidopsis thaliana (AtSRT1 and AtSRT2; AT5G55760.1, AT5G09230.7), the protozoan Trypanosoma brucei (TbSIR2rp1-3; Tb427_070021200.1

Tb427_080035900.1, Tb427_040027700.1), and the bacteria Escherichia coli (EcCobB; ANK06644.1) and Streptococcus pneumoniae (SpCobB; CWJ68461.1). The sequences were subjected to ClustalW multiple sequence alignment with the MEGAX software. An unrooted phylogenetic tree was produced by applying the neighbor-joining method with 500 bootstrap replicates.

Structural alignment of the Sir2 domain of SRTA isoforms. The 3D structures of the Sir2 domains of Chlamydomonas SRTA and the mutated versions of SRTA from UVM11 (SRTA $\mathrm{UVM}_{11}, \mathrm{P} 222 \mathrm{~L}$ ) and UVM4 (SRTA $\left.\mathrm{UVM}_{4}, \mathrm{G} 55 \mathrm{~S}\right)$ were predicted by homology modeling, based on the published 3D structure of human SIR6 (5mf6A) using ModBase (http://modbase.compbio.ucsf.edu/modbase-cgi/index. cgi). The modeled residues comprise positions 17 to 272 of the wild-type SRTA protein and amino acids 16 to 276 of the template protein SIRT6 ( $46 \%$ identity). The output structures were visualized by Chimera 1.12 (http://www.cgl.ucsf.edu/ chimera).

Validation of srta-3 and smm12 insertion mutants. Insertion mutants in loci srta (Cre10.g462200, mutant allele srta-3) and smm12 (Cre03.g200350) were ordered from the CLiP mutant library ${ }^{32}$ and the insertions were verified by PCR and Southern blot analysis. The primers used are listed in Supplementary Table 10. For Southern blotting, DNA samples from single cell-derived srta-3 mutant lines were digested with AhdI and PsiI, and hybridized to a SRTA-specific probe. A probe hybridizing to the 5 
end of the SRTA gene was generated by digesting plasmid pJR81 with ClaI and NheI, and purifying the DNA fragment of interest after gel electrophoretic separation. In addition, DNA of srta-3 mutants was digested with XhoI and HindIII, and hybridized to a probe specific for the $5^{\prime}$ end of the SMM12 gene. This probe was generated by PCR using primers oJN29 (5'-TTGCGAACACTTCGGGCGAT- $\left.3^{\prime}\right)$ and oJN30 (5'ACCACCCTGGACAGAACCGA-3'). Insertion of the $5^{\prime}$ junction of the CIB cassette into the first intron was confirmed by sequencing of the PCR amplicons. We did not succeed with mapping the exact $3^{\prime}$ junction of the insert, which was predicted to be located within gene Cre03.g200350 (SMM12) on chromosome 3. Southern blot analysis revealed an additional insertion in locus Cre03.g200350. The two insertions in Cre10g.462200 and Cre03.g200350 cannot be separated, as evidenced by analysis of T1 segregants of crosses of srta-3_1 or srta-3_2 with the wild-type CC-1690. This result suggests a complex genomic rearrangement, as it has been shown to occur frequently in insertion mutants of the CLiP library ${ }^{32,55}$. To determine SRTA transcript levels in the mutants, RT-PCR analysis was undertaken using primers SRT-E1-fw and SRT-E3-rv (Supplementary Table 10) that anneal in exon 1 and exon 3, respectively, and span the insertion site of the CIB cassette within intron 1.

As control strains, the insertion mutant LMJ.RY0402.153656 (smm12) was ordered from the CLiP library. The strain is predicted to carry an insertion of the CIB cassette in intron 6 of the SMM12 (Cre03.g200350) gene. RT-PCR analyses were performed to determine SMM12 transcript levels in the srta-3 and smm12 insertion mutants using primers oJN29 and oJN20 or oJN27 and oJN28 that anneal at the $5^{\prime}$ or $3^{\prime}$ end of the transcript, respectively (Supplementary Table 10).

Treatments with histone deacetylase inhibitors. For trichostatin inhibition of histone deacetylases, $2 \mathrm{~mL}$ cultures grown in a 24 -well plate were treated with either DMSO (as negative control) or with $200 \mathrm{ng} \mathrm{mL}^{-1}$ trichostatin (SigmaAldrich) for $20 \mathrm{~h}$. For western blot analysis $5 \mu \mathrm{g}$ of total soluble protein were loaded on SDS-polyacrylamide gels to determine YFP accumulation and the levels of acetylated histone $\mathrm{H} 3$ (H3ac).

For treatments with the inhibitor sirtinol, $2 \mathrm{~mL}$ aliquots of algal cultures grown to mid-exponential phase were transferred to a 24 -well plate and treated with sirtinol (InSolutionTM sirtinol, Merck) at final concentrations of 5, 10, and $50 \mu \mathrm{M}$ for $48 \mathrm{~h}$. Control samples were treated with DMSO only. For immunoblot analysis, $5 \mu \mathrm{g}$ of total soluble protein were loaded.

For treatments with the inhibitor OSS_128167, $1 \mathrm{~mL}$ cultures of Chlamydomonas cells were grown to mid-exponential phase in a multi-well plate and incubated with the sirtuin inhibitor OSS_128167 (Selleckchem) at a final concentration of $100 \mu \mathrm{M}$ for $48 \mathrm{~h}$. As a negative control, samples were incubated with DMSO only (i.e., $4 \mu \mathrm{l}$ of a $25 \mathrm{mM}$ OSS_128167 stock solution dissolved in DMSO, or $4 \mu \mathrm{l}$ of DMSO were added to $1 \mathrm{~mL}$ cell culture). For immunoblot analysis, samples of $15 \mu \mathrm{g}$ of extracted total soluble protein were used.

Reporting summary. Further information on research design is available in the Nature Research Reporting Summary linked to this article.

\section{Data availability}

The data supporting the findings of this study are available within the paper and its supplementary information files. A reporting summary for this article is available as a supplementary information file. The datasets and materials generated and analyzed during the current study are available from the corresponding author upon request. Highthroughput sequencing data have been deposited in the National Center for Biotechnology Information Sequence Read Archive under accession numbers SRR1797981, SRR6872092, SRR6872091, and SRR6872090 for genome resequencing of strains cw15arg-, Elow47, UVM4, and UVM11, respectively. The RNAseq data, including raw reads and FPKM expression tables, were deposited in the NCBI Gene Expression Omnibus (GEO) database under accession GSE128981. Source data are provided with this paper.

Received: 22 July 2019; Accepted: 5 November 2020;

Published online: 08 December 2020

\section{References}

1. Harris, E. H. Chlamydomonas as a model organism. Annu. Rev. Plant Physiol. Plant Mol. Biol. 52, 363-406 (2001).

2. Radakovits, R., Jinkerson, R. E., Darzins, A. \& Posewitz, M. C. Genetic engineering of algae for enhanced biofuel production. Eukaryot. Cell 9 , 486-501 (2010).

3. Scaife, M. A. et al. Establishing Chlamydomonas reinhardtii as an industrial biotechnology host. Plant J. 82, 532-546 (2015).

4. Scranton, M. A., Ostrand, J. T., Fields, F. J. \& Mayfield, S. P. Chlamydomonas as a model for biofuels and bio-products production. Plant J. 82, 523-531 (2015).

5. Gangl, D. et al. Biotechnological exploitation of microalgae. J. Exp. Bot. 66, 6975-6990 (2015).
6. Fuhrmann, M., Oertel, W. \& Hegemann, P. A synthetic gene coding for the green fluorescent protein (GFP) is a versatile reporter in Chlamydomonas reinhardtii. Plant J. 19, 353-361 (1999).

7. Schroda, M., Blöcker, D. \& Beck, C. F. The HSP70A promoter as a tool for the improved expression of transgenes in Chlamydomonas. Plant J. 21, 121-131 (2000).

8. Cerutti, H., Johnson, A. M., Gillham, N. W. \& Boynton, J. E. Epigenetic silencing of a foreign gene in nuclear transformants of Chlamydomonas. Plant Cell 9, 925-945 (1997).

9. Yamasaki, T. \& Ohama, T. Involvement of Elongin C in the spread of repressive histone modifications. Plant J. 65, 51-61 (2011).

10. Mussgnug, J. H. Genetic tools and techniques for Chlamydomonas reinhardtii. Appl. Microbiol. Biotechnol. 99, 5407-5418 (2015).

11. Schroda, M., Beck, C. F. \& Vallon, O. Sequence elements within an HSP70 promoter counteract transcriptional transgene silencing in Chlamydomonas. Plant J. 31, 445-455 (2002).

12. Lumbreras, V., Stevens, D. R. \& Purton, S. Efficient foreign gene expression in Chlamydomonas reinhardtii mediated by an endogenous intron. Plant J. 14, 441-447 (1998).

13. Shao, N. \& Bock, R. A codon-optimized luciferase from Gaussia princeps facilitates the in vivo monitoring of gene expression in the model alga Chlamydomonas reinhardtii. Curr. Genet. 53, 381-388 (2008).

14. Barahimipour, R. et al. Dissecting the contributions of GC content and codon usage to gene expression in the model alga Chlamydomonas reinhardtii. Plant J. 84, 704-717 (2015).

15. Neupert, J., Karcher, D. \& Bock, R. Generation of Chlamydomonas strains that efficiently express nuclear transgenes. Plant J. 57, 1140-1150 (2009).

16. Karcher, D., Köster, D., Schadach, A., Klevesath, A. \& Bock, R. The Chlamydomonas chloroplast HLP protein is required for nucleoid organization and genome maintenance. Mol. Plant 2, 1223-1232 (2009).

17. Lauersen, K. J., Kruse, O. \& Mussgnug, J. H. Targeted expression of nuclear transgenes in Chlamydomonas reinhardtii with a versatile, modular vector toolkit. Appl. Microbiol. Biotechnol. 99, 3491-3503 (2015).

18. Barahimipour, R., Neupert, J. \& Bock, R. Efficient expression of nuclear transgenes in the green alga Chlamydomonas: synthesis of an HIV antigen and development of a new selectable marker. Plant Mol. Biol. 90, 403-418 (2016).

19. Ramos-Martinez, E. M., Fimognari, L. \& Sakuragi, Y. High-yield secretion of recombinant proteins from the microalga Chlamydomonas reinhardtii. Plant Biotechnol. J. 15, 1214-1224 (2017).

20. Strenkert, D., Schmollinger, S., Sommer, F., Schulz-Raffelt, M. \& Schroda, M. Transcription factor-dependent chromatin remodeling at heat shock and copper-responsive promoters in Chlamydomonas reinhardtii. Plant Cell 23, 2285-2301 (2011).

21. Strenkert, D., Schmollinger, S. \& Schroda, M. Heat shock factor 1 counteracts epigenetic silencing of nuclear transgenes in Chlamydomonas reinhardtii. Nucleic Acids Res. 41, 5273-5289 (2013).

22. Lodha, M., Schulz-Raffelt, M. \& Schroda, M. A new assay for promoter analysis in Chlamydomonas reveals roles for heat shock elements and the TATA box in HSP70A promoter-mediated activation of transgene expression. Eukaryot. Cell 7, 172-176 (2008).

23. Rymarquis, L. A., Handley, J. M., Thomas, M. \& Stern, D. B. Beyond complementation. Map-based cloning in Chlamydomonas reinhardtii. Plant Physiol. 137, 557-566 (2005).

24. Pandey, R. et al. Analysis of histone acetyltransferase and histone deacetylase families of Arabidopsis thaliana suggests functional diversification of chromatin modification among multicellular eukaryotes. Nucleic Acids Res. 30, 5036-5055 (2002).

25. Bourque, S. et al. The evolution of HD2 proteins in green plants. Trends Plant Sci. 21, 1008-1016 (2016).

26. Frye, R. A. Phylogenetic classification of prokaryotic and eukaryotic Sir2-like proteins. Biochem. Biophys. Res. Commun. 273, 793-798 (2000).

27. Michishita, E. et al. SIRT6 is a histone H3 lysine 9 deacetylase that modulates telomeric chromatin. Nature 452, 492-496 (2008).

28. Tong, Z. et al. SIRT7 is activated by DNA and deacetylates histone $\mathrm{H} 3$ in the chromatin context. ACS Chem. Biol. 11, 742-747 (2016).

29. Jiang, H. et al. SIRT6 regulates TNF- secretion through hydrolysis of long chain fatty acyl lysine. Nature 496, 110-113 (2013).

30. Tao, N.-N. et al. Deacetylation of Ku70 by SIRT6 attenuates Bax-mediated apoptosis in hepatocellular carcinoma. Biochem. Biophys. Res. Commun. 485, 713-719 (2017)

31. Chen, S. et al. Repression of RNA polymerase I upon stress is caused by inhibition of RNA-dependent deacetylation of PAF53 by SIRT7. Mol. Cell 52, 303-313 (2013)

32. Li, X. et al. An indexed, mapped mutant library enables reverse genetics studies of biological processes in Chlamydomonas reinhardtii. Plant Cell 28, 367-387 (2016).

33. Grozinger, C. M., Chao, E. D., Blackwell, H. E., Moazed, D. \& Schreiber, S. L. Identification of a class of small molecule inhibitors of the sirtuin family of 
NAD-dependent deacetylases by phenotypic screening. J. Biol. Chem. 276, 38837-38843 (2001).

34. Mai, A. et al. Design, synthesis, and biological evaluation of sirtinol analogues as class III histone/protein deacetylase (Sirtuin) inhibitors. J. Med. Chem. 48, 7789-7795 (2005).

35. Parenti, M. D. et al. Discovery of novel and selective SIRT6 inhibitors. J. Med. Chem. 57, 4796-4804 (2014).

36. Merchant, S. S. et al. The Chlamydomonas genome reveals the evolution of key animal and plant functions. Science 318, 245-251 (2007).

37. Wu-Scharf, D., Jeong, B.-r, Zhang, C. \& Cerutti, H. Transgene and transposon silencing in Chlamydomonas reinhardtii by a DEAH-box RNA helicase. Science 290, 1159-1162 (2000).

38. Pontier, D. et al. Reinforcement of silencing at transposons and highly repeated sequences requires the concerted action of two distinct RNA polymerases IV in Arabidopsis. Genes Dev. 19, 2030-2040 (2005).

39. Tanny, J. C., Dowd, G. J., Huang, J., Hilz, H. \& Moazed, D. An enzymatic activity in the yeast Sir2 protein that is essential for gene silencing. Cell 99, 735-745 (1999).

40. Kawahara, T. L. et al. Dynamic chromatin localization of Sirt6 shapes stressand aging-related transcriptional networks. PLoS Genet. 7, e1002153 (2011).

41. Bock, R. The give-and-take of DNA: horizontal gene transfer in plants. Trends Plant Sci. 15, 11-22 (2010).

42. Wommack, K. E. \& Colwell, R. R. Virioplankton: viruses in aquatic ecosystems. Microbiol. Mol. Biol. Rev. 64, 69-114 (2000).

43. Van Etten, J. L., Lane, L. C. \& Meints, R. H. Viruses and viruslike particles of eukaryotic algae. Mcriobiol. Rev. 55, 586-620 (1991).

44. Harris, E. H. The Chlamydomonas Sourcebook. (Academic Press, San Diego, CA, 1989).

45. Kropat, J. et al. A revised mineral nutrient supplement increases biomass and growth rate in Chlamydomonas reinhardtii. Plant J. 66, 770-780 (2011).

46. Berthold, P., Schmitt, R. \& Mages, W. An engineered Streptomyces hygroscopicus aph7" gene mediates dominant resistance against hygromycin B in Chlamydomonas reinhardtii. Protist 153, 401-412 (2002).

47. Kindle, K. L., Richards, K. L. \& Stern, D. B. Engineering the chloroplast genome: Techniques and capabilities for chloroplast transformation in Chlamydomonas reinhardtii. Proc. Natl Acad. Sci. USA 88, 1721-1725 (1991).

48. Neupert, J., Shao, N., Lu, Y. \& Bock, R. Genetic transformation of the model green alga Chlamydomonas reinhardtii. Meth. Mol. Biol. 847, 35-47 (2012).

49. Strenkert, D., Schmollinger, S. \& Schroda, M. Protocol: methodology for chromatin immunoprecipitation (ChlP) in Chlamydomonas reinhardtii. Plant Methods 7, 35 (2011).

50. Gallaher, S. D., Fitz-Gibbon, S. T., Glaesener, A. G., Pellegrini, M. \& Merchant, S. S. Chlamydomonas genome resource for laboratory strains reveals a mosaic of sequence variation, identifies true strain histories, and enables strainspecific studies. Plant Cell 27, 2335-2352 (2015).

51. Li, H. \& Durbin, R. Fast and accurate short read alignment with BurrowsWheeler transform. Bioinformatics 25, 1754-1760 (2009).

52. Cingolani, P. et al. A program for annotating and predicting the effects of single nucleotide polymorphisms, SnpEff: SNPs in the genome of Drosophila melanogaster strain w1118; iso-2; iso-3. Fly 6, 80-92 (2012).

53. Zhu, A., Ibrahim, J. G. \& Love, M. I. Heavy-tailed prior distributions for sequence count data: removing the noise and preserving large differences. Bioinformatics 35, 2084-2092 (2019).

54. Larkin, M. A. et al. Clustal W and Clustal X version 2.0. Bioinformatics 23 , 2947-2948 (2007).
55. Chaux, F. et al. Flavodiiron proteins promote fast and transient $\mathrm{O} 2$ photoreduction in Chlamydomonas. Plant Physiol. 174, 1825-1836 (2017).

\section{Acknowledgements}

We thank Richard Yu, Gustavo Pesce, and the former staff at Green Pacific Biologicals Inc. for help with genetic crosses. This work used the Vincent J. Coates Genomics Sequencing Laboratory at UC Berkeley, supported by NIH S10 OD018174 Instrumentation Grant. This research was financed by the Max Planck Society. S.D.G. and S.F.G. were supported by the U.S. Department of Energy (Grant DE-FC02-02ER63421).

\section{Author contributions}

J.N., S.D.G., Y.L., D.S., N.S., R. Barahimipour, and S.T.F.-G. performed the experiments All authors participated in data evaluation and experimental design. R. Bock conceived of the study, and wrote the manuscript together with J.N.

\section{Funding}

Open Access funding enabled and organized by Projekt DEAL.

\section{Competing interests}

The expression strains used in this study are covered by a patent application (PCT/ EP2009/003684 with J.N. and R.Bo. as inventors) and are available under a Material Transfer Agreement. All other authors declare no competing interests.

\section{Additional information}

Supplementary information is available for this paper at https://doi.org/10.1038/s41467 020-19983-4.

Correspondence and requests for materials should be addressed to R. Bock.

Peer review information Nature Communications thanks Robert Jinkerson, Attila Molnar and the other, anonymous, reviewer(s) for their contribution to the peer review of this work. Peer reviewer reports are available.

Reprints and permission information is available at http://www.nature.com/reprints

Publisher's note Springer Nature remains neutral with regard to jurisdictional claims in published maps and institutional affiliations.

Open Access This article is licensed under a Creative Commons Attribution 4.0 International License, which permits use, sharing, adaptation, distribution and reproduction in any medium or format, as long as you give appropriate credit to the original author(s) and the source, provide a link to the Creative Commons license, and indicate if changes were made. The images or other third party material in this article are included in the article's Creative Commons license, unless indicated otherwise in a credit line to the material. If material is not included in the article's Creative Commons license and your intended use is not permitted by statutory regulation or exceeds the permitted use, you will need to obtain permission directly from the copyright holder. To view a copy of this license, visit http://creativecommons.org/ licenses/by/4.0/

(c) The Author(s) 2020, corrected publication 2021 